\title{
Fault Severity Evaluation and Improvement Design for Mechanical Systems Using the Fault Injection Technique and Gini Concordance Measure
}

\author{
Jianing $W u$ and Shaoze Yan \\ State Key Laboratory of Tribology, Department of Mechanical Engineering, Tsinghua University, Beijing 100084, China \\ Correspondence should be addressed to Shaoze Yan; yansz@tsinghua.edu.cn
}

Received 23 October 2013; Accepted 4 January 2014; Published 29 April 2014

Academic Editor: G. Rega

Copyright (C) 2014 J. Wu and S. Yan. This is an open access article distributed under the Creative Commons Attribution License, which permits unrestricted use, distribution, and reproduction in any medium, provided the original work is properly cited.

\begin{abstract}
A new fault injection and Gini concordance based method has been developed for fault severity analysis for multibody mechanical systems concerning their dynamic properties. The fault tree analysis (FTA) is employed to roughly identify the faults needed to be considered. According to constitution of the mechanical system, the dynamic properties can be achieved by solving the equations that include many types of faults which are injected by using the fault injection technique. Then, the Gini concordance is used to measure the correspondence between the performance with faults and under normal operation thereby providing useful hints of severity ranking in subsystems for reliability design. One numerical example and a series of experiments are provided to illustrate the application of the new method. The results indicate that the proposed method can accurately model the faults and receive the correct information of fault severity. Some strategies are also proposed for reliability improvement of the spacecraft solar array.
\end{abstract}

\section{Introduction}

Nowadays, high reliability performance is required for mechanical systems, motivating scholars to develop a variety of methods and strategies to improve the performance of the mechanical systems [1-4], from conceptual design stage when information is insufficient compared to the detailed design phrase when adequate empirical data are collected [15].

Till now, three types of reliability analysis for mechanical systems have been proposed. The first type of methods originates from the fault tree analysis (FTA) and reliability block diagram (RBD) [6]. For instance, Xiao et al. [7] discussed the reliability modeling and analysis method of one typical mechanical system based on FTA and summarized the possible faults and their mechanism. Furthermore, FTA and RBD have been proved to be effective in summarizing the faults in the initial stage of reliability analysis [7]. The second way is proposed by using the extended form of Petri net and the neutral network [8-13]. For example, many extensions to the Petri nets, such as the colored Petri net, timed Petri net, and prioritized Petri net, have been successfully developed and applied in analyzing working properties of systems and conducting fault diagnosis [8-10]. A major concern in enhancing reliability of the mechanical systems today is to continue improving their dynamic performance. These methods listed in [8-13] are verified effectively in reliability analysis of the distributed mechanical systems, but they did not consider more on whether the components are in good condition or not, rather than the dynamic performance. The third type is conducted, within the scope of dynamic properties, applying the numerical simulation to measure the dynamic properties of the mechanical system with faults and detecting the failure mechanism and weak links of the system $[14,15]$. But the methodologies are not developed and little literature related to this area has been published.

Although some progress has been made in this area, at least four major barriers must be removed to perfectly realize the reliability analysis of the mechanical systems. Firstly and obviously, for a long time it has been wellknown that reliability, availability, and safety of complicated mechanical systems cannot be obtained solely by the careful design, the quality endurance of manufacturing, or other techniques for preventing failure. This might be caused 
by the properties inherent to the system itself and the complex relationship between the units and unknown fault characteristics embedded in the system. It is verified that the complicated connections of the components cannot be described by the Boolean logical symbols. Namely, FTA and RBD cannot model the mechanical system accurately. Moreover, the conventional methods of analyzing reliability rely on the statistical models. The use of statistical-based methodologies, partly effective though, tends to be not so easy for reliability improvement on account of lack of failure data. Take the typical mechanical system, that is, the spacecraft solar array, as an example $[1,7]$. Over the last ten years, Airclaim's Ascend SpaceTrak database has documented 117 satellites solar array anomalies, in which twelve solar array anomalies directly resulted in total satellite failure. Designers have well-recognized the importance of solar array reliability, but to our best knowledge a detailed reliability analysis is still lacking $[16,17]$. So it is of great importance to investigate the fault severity of the spacecraft solar array. As it is impossible to wait and get statistical data from field operation of the system for one spacecraft design task, it is necessary that preliminary studies of the dynamic operation of the mechanical systems in presence of anticipated faults should be figured out during all phases of operation. It is quite evident that seizing the weak links of the mechanical system should be a better way to evaluate and prompt the reliability of the mechanical system. Secondly, the approach considering dynamic properties is probably much more useful for actual engineering applications. There is considerable amount of literature on investigating the characteristics of the dynamic output with many types of faults, such as the acceleration, momentum, and kinetic energy. It is worth noting that this type of methods focuses on the dynamic property which is the symbol of performance for a mechanical system. But the evaluation methods, including the approach of capturing the severity of the faults, are not clearly identified. Namely, in the field of dynamic characteristic based methodologies, the indicators which reveal the uniqueness of diverse faults have been scarcely reported. Thirdly, some scholars simulated the dynamical performance of the typical mechanical systems with faults $[14,15]$, yet little methodologies have been carried out in the scope of experiments to validate the results and seek the fault-prone parts. Finally, it is well-recognized that improving the weak links of the system is more meaningful than promoting all of the parts in the system. So in another view, the severity ranking of the units in the subsystems is the premise of reliability improvement. We can summarize that ranking of the faults can be roughly obtained by the existing methodologies, but the quantitative and credible ranking of components could not be clearly identified.

To sum up, a major thrust of reliability analysis for complex mechanical systems is to get the weak links of the mechanical system by the dynamic properties and to acquire the information of the components which is easier to fail. Namely, completing the fault severity evaluation is a more effective way of reliability analysis for mechanical systems. As a result, the concept of combining the fault injection techniques and the Gini concordance for fault severity ranking evaluation as a new approach to realize fault ranking is introduced in this paper.

Fault injection is the notion firstly raised by the computer scientists that is defined as the reliability validation technique on the basis of the realization of the system [18]. That is, the artificial faults are injected into the system and the resulting behavior is observed. The earliest work done for fault injection can be traced back to Harlan Mill's (IBM) work [19-21]. Attempts to resolve the problem of low dependability in computer system have resulted in the development of fault injection. In the 1980s when the integrated circuit (IC) was rapidly developed, the hardware-based fault injection was widely employed to exercise a system under the analysis with specially designed test hardware [21]. A couple of methods related to fault injection were proposed to solve issues related to reliability analysis and a wealth of information can be found in the related literature $[21,22]$. Fault removal and fault forecasting can be carried out by the fault injection techniques which yield the following three benefits [21]. The first is that it helps understand the effects of the real faults and the related behavior of the target system. The second is that it provides a feedback for enhancement and correction of the system. The third is that it forecasts the faulty behavior of the target system. From the literature survey, fault injection is an effective way to investigate the reliability of the mechanism, but the concept and application of fault injection have been rarely applied to fault analysis of the mechanical system, which is known as the multibody dynamic systems. So this paper tries to obtain the working properties with faults by using the fault injection techniques.

After we get the output dynamic parameters with faults using the technique of fault injection, one indicator is needed to evaluate the severity of the faults. The traditional methods, such as the evaluation of the relative error, strongly require the discrete data with same amount which might not be always satisfied. In addition, the concordance reflecting the trend of the data may not be clearly rated by the tradition relative error. In the 1910s, Corrado Gini introduced a measure of association named Gini concordance, which is widely used in modern economics and management [23]. The literature shows that the Gini concordance presents the accurate evaluation of the dependence among the parameters, quantitatively exploring the intrinsic relations of them [23-26]. What makes Gini concordance measure better for fault severity analysis is that it has the concrete mathematical description and clear physical meaning which is adaptive for measuring the severity of different faults, especially fits for processing the data of dynamic output. Especially, the Gini concordance can compare the trend of the two couples of data of dynamic properties. Since then, reliability approach by using Gini concordance has been extensively explored and it is still under investigation as well in methodological aspects as in concrete applications.

Consequently, the primary purpose of this paper is to implement the research on fault severity evaluation by using the fault injection techniques to propose the index, to evaluate the severity of faults, and to explore the characteristic and uniqueness of the faults embedded in the mechanical system. The concept and technique of fault injection are 
used to get the dynamic behavior of the mechanical system. The Gini concordance is applied to measure the severity of different faults. The methodologies are illustrated by one case study of the spacecraft solar array mechanism. Overall, this paper is structured as follows. Section 2 devotes to the proposed method involving the fault injection technique for mechanical system and the formulation of Gini concordance. Section 3 selects the spacecraft solar array as a case to validate this new method. In this section, the dynamic performance with local faults by fault injection technique and the severity ranking of the faults by the Gini concordance are investigated. All the results are demonstrated by the experiment conducted on the setup. This study illustrates the weak links of the solar array and gives the strategies for reliability design. The last section concludes the paper.

\section{The Proposed Method}

The new method consists of two aspects, the technique fault injection technique and the severity ranking by Gini concordance. The ranking of fault severity can be achieved by the following steps (Figure 1).

(1) The fault modes are investigated in this step. For example, some basic fault modes such as crack, deadlocking, and lack of driving force can be summarized by the fault tree analysis (FTA) methods.

(2) The selected fault modes can be injected into the model and the prototype by the simulation-based technique and the execution-based technique, respectively. Mathematical modeling and solution are processed in this step. The correctness of the simulation model is validated by the dynamic output generated from the prototype. Then, all kinds of diverse output reflecting the dynamic characteristics of the mechanical system can be obtained.

(3) Gini concordance is used to evaluate the dependence between the normal and abnormal operation and to judge the severity of the faults. As depicted in Figure 1, in the table of summary of Gini concordance, Abnormal_1 is less severe than Abnormal_2. The reliability strategies should incline to adopt some techniques to improve the reliability of the related parts in Abnormal_2.

The following parts in this section provide detailed illustration of the new method.

2.1. Fault Mode Analysis. At the beginning, designers should determine the types of faults which will be injected to the system. The approach to filtering the faults can be summarized in the following steps.

(1) System Decomposition. In order to analyze working principle of the studied mechanical system, the system can be decomposed into many subsystems and components based on the fault logic (Figure 2(a)).

(2) Establishment of the FTA Model. To understand the logic leading to the top event, one can get the FTA model by the events and logic gates $[6,27]$. By analyzing the relations between all of the parts in the mechanism, one can connect the components/events by the AND gate or OR gate. Some tests can be conducted to validate the correctness of the FTA model. Figure 2 shows the crank slider mechanism as an example to illustrate the new method of fault injection. The mechanism can be separated into the motor, the crank, the link and the slider, and the sensors. All of these parts can be connected by the logical gates of AND, OR, or NOT. Take the fault tree in Figure 2(b) for instance; the FT illustrates the law of all the connections in the system. The FT can be summarized into four layers from the total system, main faults to the subsystems, and the units/components. The notation $T$ denotes the top event and $X_{i}\left(i=1,2, \ldots, n_{b}\right)$ represents the $i$ th $\left(i=1,2, \ldots, n_{b}\right)$ bottom event in which $n$ is the number of the bottom events. The top event can be expressed by the Boolean algebra function $f$ using the logic symbols AND and OR:

$$
T=f\left(X_{i}\right) \quad\left(i=1,2, \ldots, n_{b}\right) .
$$

So the probability of the top event described as the probability of the system fault is stated as

$$
P=P(T) \quad\left(i=1,2, \ldots, n_{b}\right) .
$$

In this case, reliability degree of the system equals to

$$
R(T)=1-P(T) .
$$

(3) Identification of the Fault Probability. Concerning the experts' experiences and the history data, the Delphi method is used to provide the fault probability of the faults [28]. Table 1 collects the judgments of different experts with the score scale $[0,10] . N$ experts are invited to evaluate the $n$ bottom events by providing scores; the score of the $i$ th event can be achieved as follows:

$$
\theta_{i}=\frac{A_{i} \sum_{j=1}^{N} S_{j i}}{10 N} \quad(i=1,2, \ldots, n),
$$

where $S_{j i}$ is the score of the $i$ th bottom event provided by $j$ th $(j=1,2, \ldots, N)$ expert. $A_{i}(i=1,2, \ldots, n)\left(A_{i} \geq 1\right)$ is the adjustment parameter of the $i$ th bottom events which is derived from the previous operation data. If the event of the $i$ th bottom events did not happen, then $A_{i}=1$. If the event could be searched as actual cases in the previous data, then $A_{i}>1$. We define that if it happened once, then $A_{i}=1.1$, twice, then $A_{i}=1.2$, three times then $A_{i}=1.3$, and so forth.

According to $\theta_{i}$, one can search the corresponding fault probabilities of the different events as $p_{i}(i=1,2, \ldots, n)$ in Table 2, in which ppm means parts per million [6].

(4) Determination of the Injected Faults. The probability importance reveals the criticality of the components: 


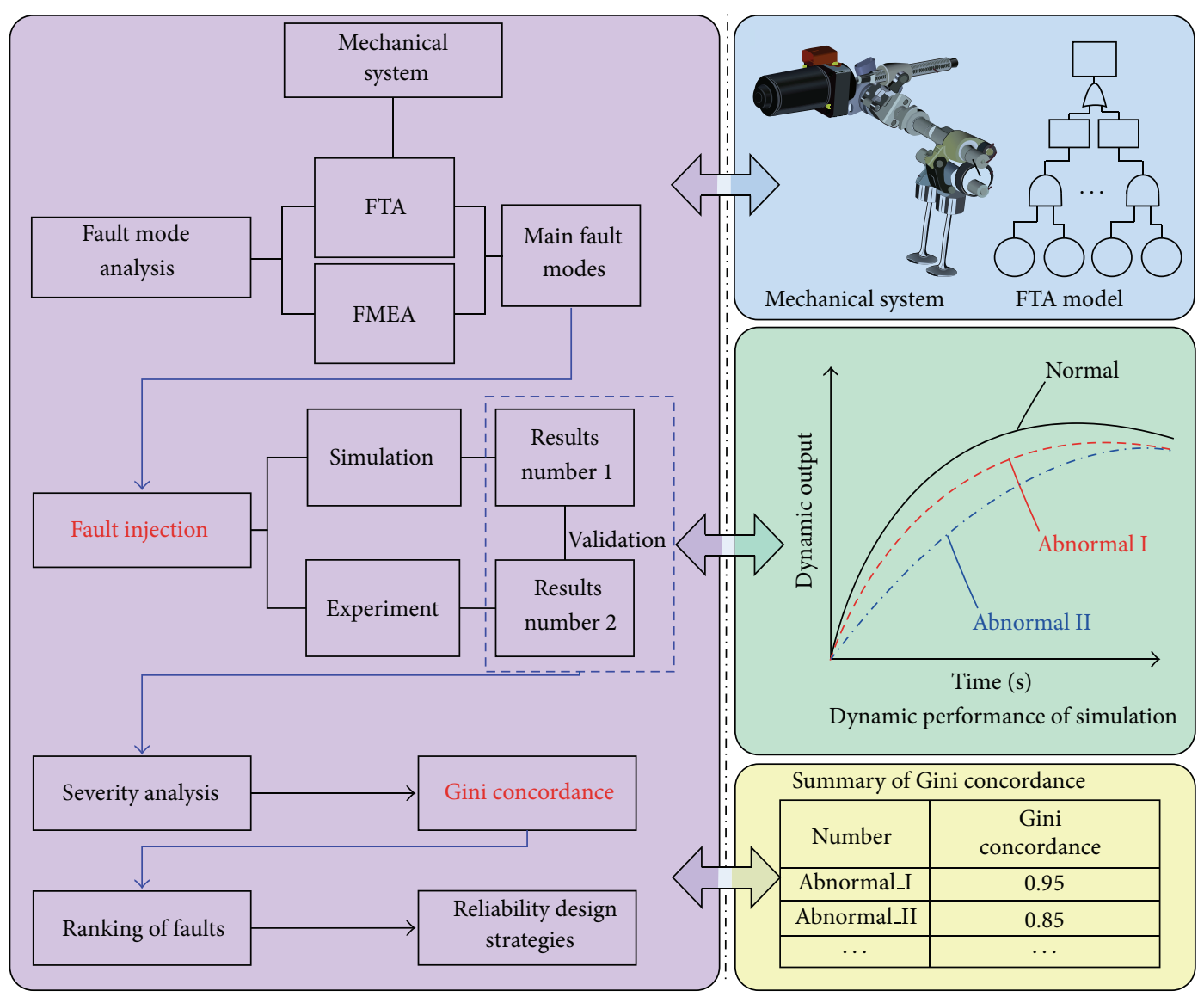

FIGURE 1: Flow chart of the proposed methods for fault severity evaluation.

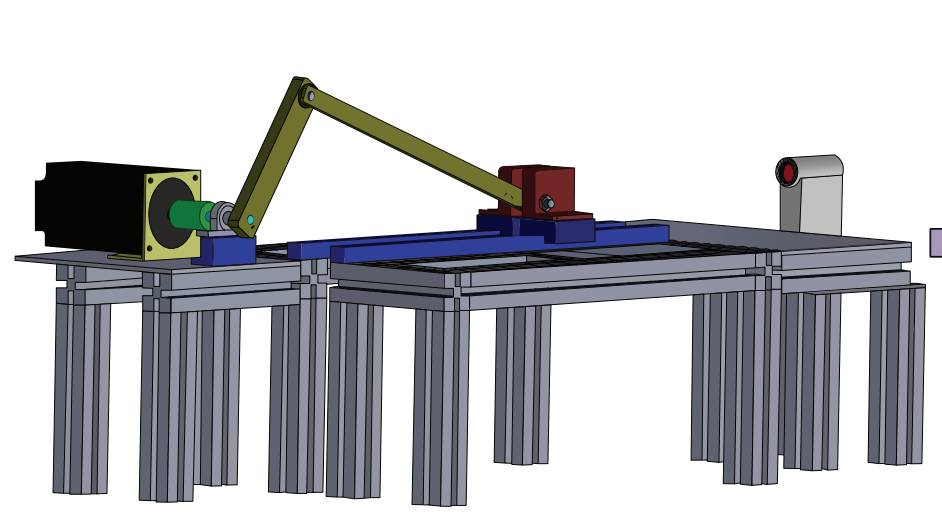

(a) Mechanical system

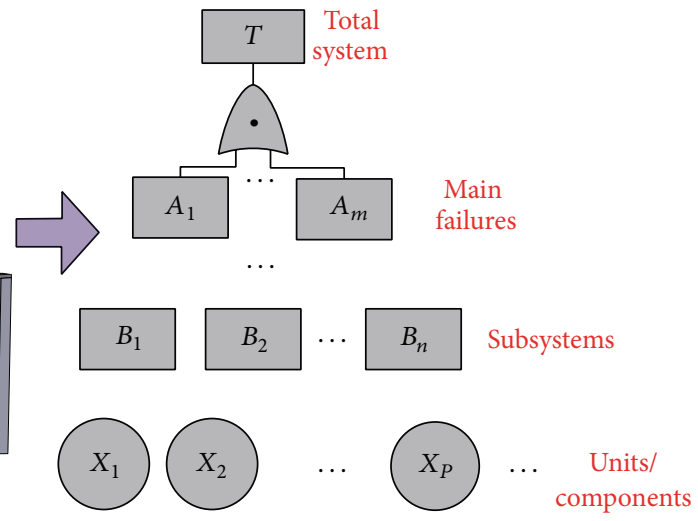

(b) FTA model

Figure 2: One mechanical system and its FTA model.

the higher the value is, the more important the fault is. This index is defined as [29]

$$
I_{h}(j)=\frac{\partial f}{\partial X_{i}}
$$

Referring to the results of the probability importance, the faults which need to be injected to the system are determined [29]. According to the evaluation results from the probability importance, one can initially and roughly judge which faults are relatively dangerous. The fault which owns higher probability importance is easier to fail.

\subsection{Fault Injection Technique and Dynamic Output. As} reported, there are two major categories of fault injection techniques: the simulation-based and execution-based [21]. In the former category, a model of one system is developed 
TABLE 1: Collection table of Delphi method.

\begin{tabular}{lcccccc}
\hline Score scale: $0 \sim 10$ & Expert I & Expert II & Expert III & $\cdots$ & Expert $N$ & Adjustment parameter \\
\hline Bottom event 1 & $S_{11}$ & $S_{21}$ & $S_{31}$ & $\cdots$ & $S_{N 1}$ & $A_{1}$ \\
Bottom event 2 & $S_{12}$ & $S_{22}$ & $S_{32}$ & $\cdots$ & & $S_{22}$ \\
$\vdots$ & & & & & $S_{N i}$ & $A_{i}$ \\
Bottom event $i$ & $S_{1 i}$ & $S_{2 i}$ & $S_{3 i}$ & $\cdots$ & & $A_{n}$ \\
$\vdots$ & & & & & & $S_{N n}$ \\
Bottom event $n$ & $S_{1 n}$ & $S_{2 n}$ & $S_{3 n}$ & $\cdots$ & & \\
\hline
\end{tabular}

TABLE 2: Assessment criterion of the fault probability.

\begin{tabular}{lc}
\hline$\theta_{i}$ & Fault probablity $p_{i}(\mathrm{ppm})$ \\
\hline 1 & 1 \\
2 & 50 \\
3 & 100 \\
4 & 500 \\
5 & 1000 \\
6 & 5000 \\
7 & 10000 \\
8 & 50000 \\
9 & 100000 \\
10 & 500000 \\
\hline
\end{tabular}

and faults are introduced. The model is then simulated to investigate the effects of the fault on the operation of the system. In the latter one, the system itself is manufactured as a prototype and some rules are used to cause typical faults in the system, and the dynamic results reflect the severity of the consequences. Additionally, the weak links can be successfully found. The execution-based method validates the results of simulation-based method. Two points should be highlighted here. Firstly, we focused on the dynamic problems of one mechanical system, so the fault injection technique is indeed operated by changing the dynamical parameters. Secondly, it should be noted that the failure mechanism of the mechanical system is always "binary," which means that the main means of the fault injection is completed by removing the related parts in the mechanical system.

2.2.1. The Simulation-Based Technique. The simulation-based technique of mechanical system needs one complete description of the dynamic behavior, namely, the dynamic equations. Generally, consider a mechanical system which can be described by the standard holonomic form of the Lagrange's equation:

$$
\frac{d}{d t}\left(\frac{\partial L}{\partial \dot{q}}\right)-\frac{\partial L}{\partial q}=0 \quad(i=1,2, \ldots, n)
$$

where $n$ is the number of coordinates and $q$ is the generalized coordinates. $T$ and $V$ represent the kinetic energy and the potential energy of the system. $L$ is the Lagrangian function as

$$
L(q, \dot{q}, t)=T(q, \dot{q}, t)=-V(q, t) .
$$

As the mechanism illustrated in Figure 3(a), particles with the masses $m_{1}$ and $m_{2}$ are connected by a rigid massless rod. The spring with stiffness $K$ and damper with damping ratio $\mu$ link $m_{2}$ and the rod and then the rod rotates around the point $O$ with the angular velocity $\omega$.

For this mechanical system, the faults can be injected by the following techniques.

(1) Mass. The injection law of mass fits for the condition that the component is lost by reasons such as the fault of fracture. Comparing Figure 3(b) with Figure 3(a), the fault will be injected by setting the mass as

$$
m_{i}=0 \quad\left(i=1,2, \ldots, n_{m}\right),
$$

where $n_{m}$ is the number of the masses in the system.

(2) Rotational Inertia. For the rotational components, if the component is out of service, the fault can be injected by setting the rotational inertia as

$$
I_{i}=0 \quad\left(i=1,2, \ldots, n_{I}\right),
$$

where $n_{I}$ is the number of the related rotational inertias in the system.

(3) Stiffness. The compressive spring and the torsion spring are widely used in mechanical systems. Figure 3(c) illustrates the method of fault injection for this kind of fault. If the spring is out of service, the fault will be injected by setting the stiffness as

$$
\begin{aligned}
& K_{C i}=0, \quad \text { compressive spring } \\
& K_{T i}=0, \quad \text { torsion spring } \\
& \quad\left(i=1,2, \ldots, n_{s}\right),
\end{aligned}
$$

where $n_{s}$ is the number of the compressive or torsion springs.

(4) Damper. If the damper fails, the fault will be injected by setting the stiffness as shown in Figure 3(d) as

$$
\mu=0 \quad\left(i=1,2, \ldots, n_{\mu}\right),
$$

where $n_{\mu}$ is the number of the dampers. 


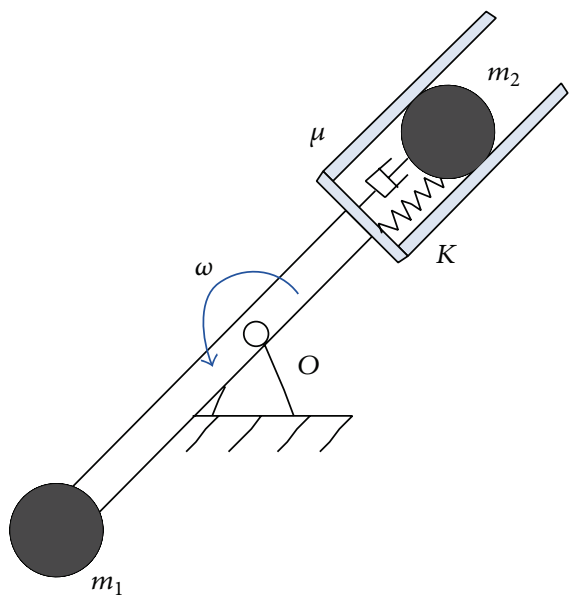

(a)

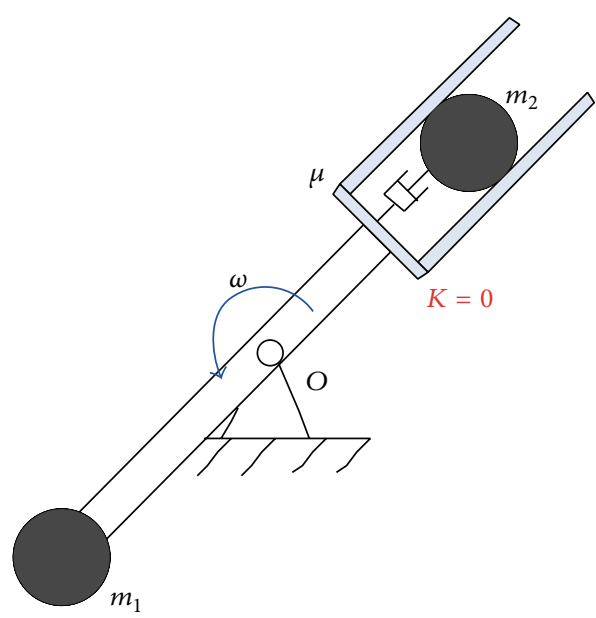

(c)

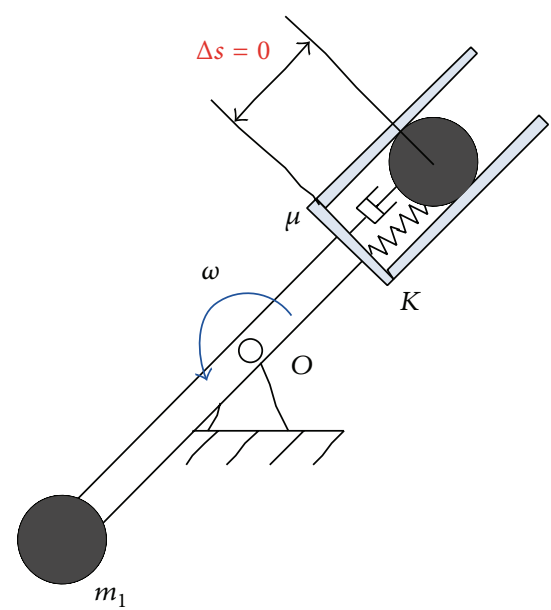

(e)

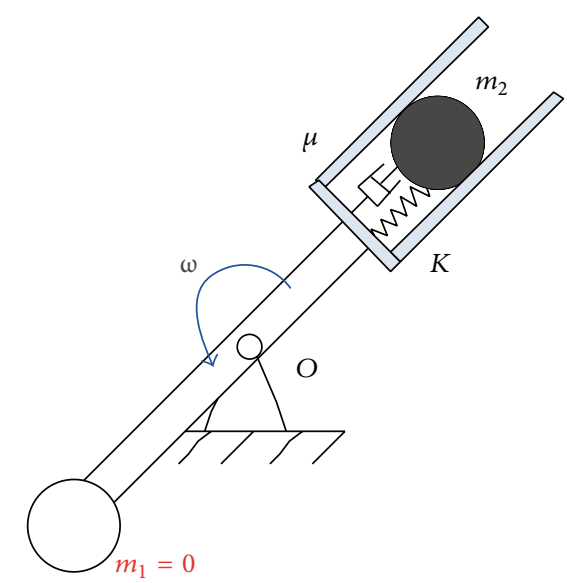

(b)

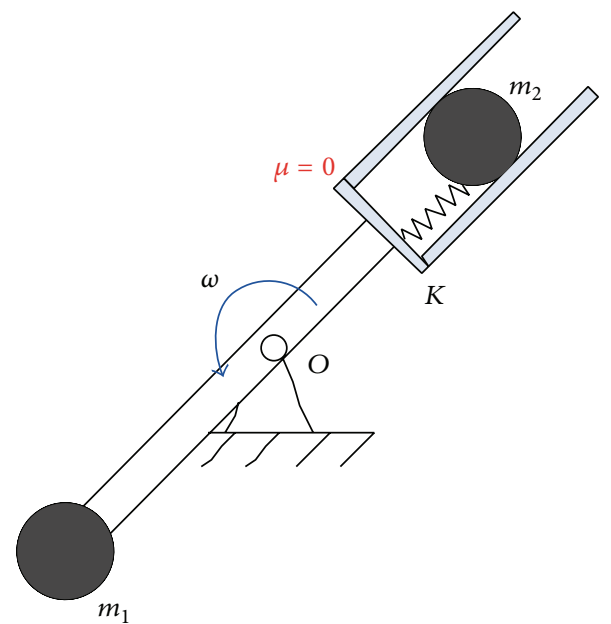

(d)

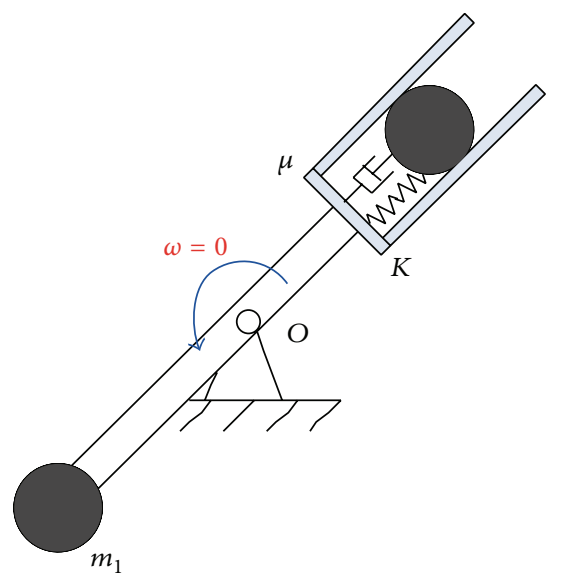

(f)

Figure 3: Examples of simulation-based technique for fault injection. 


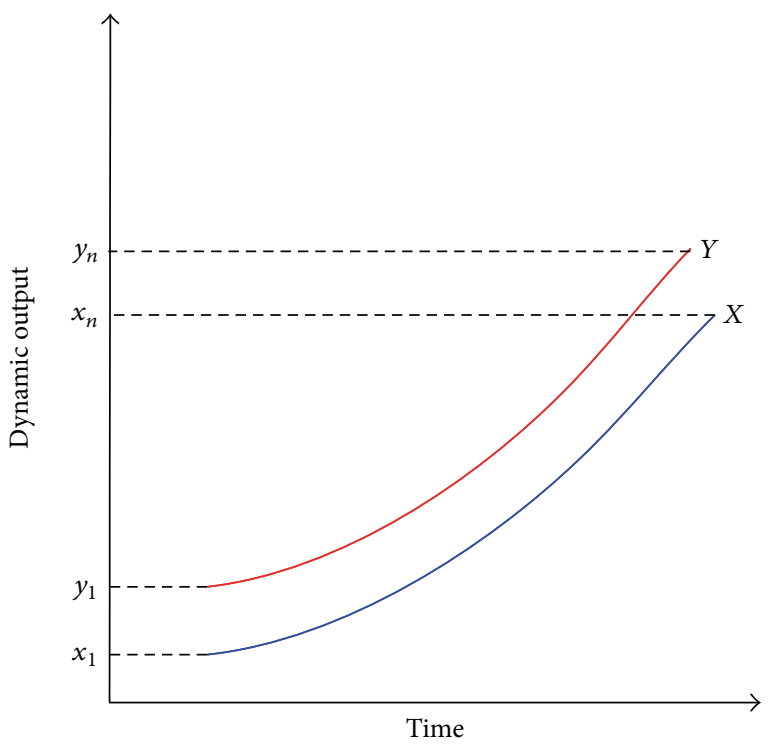

(a)

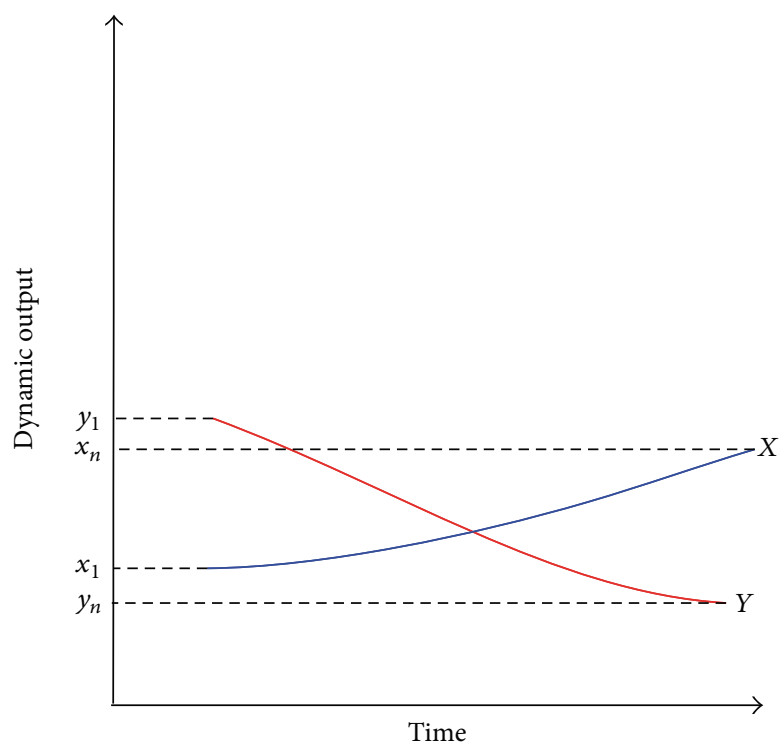

(b)

FIgure 4: Two time domain vectors.

(5) Deadlocking of the Slider. As depicted in Figure 3(e), if the slider is stuck, the fault will be injected by setting the relative displacement as

$$
\Delta s=0 \quad\left(i=1,2, \ldots, n_{d}\right),
$$

where $n_{d}$ denotes the number of the sliders.

(6) Fault of the Drive Motor. As shown in Figure 3(f), if the motor fails, the input axis will be fixed and the angular velocity will be set as

$$
\omega=0 \quad\left(i=1,2, \ldots, n_{\omega}\right),
$$

where $n_{\omega}$ denotes the number of the drive motors.

2.2.2. The Execution-Based Technique. The execution-based technique for fault injection is conducted in an actual experimental setup or a prototype [21]. Considering the most widely used machines, the techniques for fault injection are summarized in Table 3.

2.2.3. Dynamic Output. According to the analytical resultsbased method, the dynamic output will be obtained by solving the dynamic equations. For instance, the dynamic output of the normal and abnormal operation can be described as the vectors $\mathbf{X}$ and $\mathbf{Y}$ :

$$
\mathbf{X}=\left\{x_{i}\right\}_{i=1,2, \ldots, n}, \quad \mathbf{Y}=\left\{y_{i}\right\}_{i=1,2, \ldots, n},
$$

where $n$ is the size of the output vectors.

2.3. Formulation of Gini Concordance. Generally a main idea of fault severity analysis is to calculate the degree of similarity between the ideal dynamic output and the faulty output.

\begin{tabular}{|c|c|}
\hline Fault & Fault injection method \\
\hline Loss of mass & Remove the related components \\
\hline Fault of the spring(s) & Remove the spring(s) \\
\hline Fault of the damper(s) & Remove the damper(s) \\
\hline $\begin{array}{l}\text { Fault of the drive } \\
\text { motor(s) }\end{array}$ & Remove the motors(s) \\
\hline $\begin{array}{l}\text { Lack of torque in the } \\
\text { spring(s) }\end{array}$ & $\begin{array}{l}\text { Change the spring(s) into a smaller one } \\
\text { or remove the spring(s) }\end{array}$ \\
\hline $\begin{array}{l}\text { Fault of locking } \\
\text { mechanism }\end{array}$ & Remove the locking spring(s) \\
\hline $\begin{array}{l}\text { Fault of the drive } \\
\text { motor }\end{array}$ & $\begin{array}{l}\text { Turn off the drive motor and fixed the } \\
\text { shaft }\end{array}$ \\
\hline Fault of deadlocking & Fix the slider, joints, or hinges by the plug \\
\hline
\end{tabular}
The traditional formulation denotes the average absolute
TABLE 3: Execution-based techniques for fault injection.

difference between the two vectors. This index is able to reveal the actual discrepancy of the two vectors, but it cannot distinguish the trend of the varying variables. As in the case shown in Figure 4, the average discrepancies between $X$ and $Y$ in (a) and (b) are probably similar, whereas their trends are distinct to each other. If we use the conventional methodologies of actual discrepancy of the two vectors, these two cases may get the same concordance measure. In order to comprehensively take account of the data concordance, the Gini concordance measure is introduced to evaluate the fault severity.

The Gini concordance is adopted in evaluating the severity of the fault caused by particular links. On the basis of (14) two ascending sequences are depicted as [19]

$$
x_{(1)}<x_{(2)}<\cdots x_{(n)}, \quad y_{(1)}<y_{(2)}<\cdots y_{(n)} .
$$


The vectors $p=\left\{p_{i}\right\}_{i=1,2, \ldots, n}$ and $q=\left\{q_{i}\right\}_{i=1,2, \ldots, n}$ represent the ranks in a sample of size $n$ of two continuous random variables $\mathbf{X}$ and $\mathbf{Y}$. Then, the Gini concordance $\gamma$ can be defined as [21]

$$
\gamma=\frac{1}{\left\lfloor n^{2} / 2\right\rfloor}\left[\sum_{i=1}^{n}\left|p_{i}+q_{i}-n-1\right|-\sum_{i=1}^{n}\left|p_{i}-q_{i}\right|\right],
$$

where $\left\lfloor n^{2} / 2\right\rfloor$ denotes the integer part of $\left(n^{2} / 2\right)$.

2.4. Effective Period and Data Unity. The dynamic output is summarized as the displacement or the angular displacement $\Theta=\left(\Theta_{1}, \Theta_{2}, \ldots, \Theta_{n}\right)_{1 \times n}$ which can be directly detected by the sensors on the uniform time interval $\Delta t$. Then, the (angular) velocity $\dot{\Theta}=\left(\dot{\Theta}_{1}, \dot{\Theta}_{2}, \ldots, \dot{\Theta}_{n}\right)_{1 \times n}$ and the (angular) acceleration $\ddot{\Theta}=\left(\ddot{\Theta}_{1}, \ddot{\Theta}_{2}, \ldots, \ddot{\Theta}_{n}\right)_{1 \times n}$ can be derived and implemented by the differential method as

$$
\begin{aligned}
& \dot{\Theta}_{1}=\frac{\left(\Theta_{2}-\Theta_{1}\right)}{\Delta t}, \\
& \dot{\Theta}_{k}=\frac{\left(\Theta_{k}-\Theta_{k-1}\right)}{\Delta t}, \\
& \ddot{\Theta}_{1}=\frac{\left(\dot{\Theta}_{2}-\dot{\Theta}_{1}\right)}{\Delta t}, \\
& \ddot{\Theta}_{k+1}=\frac{\left(\dot{\Theta}_{k+1}-\dot{\Theta}_{k}\right)}{\Delta t}, \\
& (k=2,3, \ldots, n) .
\end{aligned}
$$

Two key issues must be clearly identified before the fault severity evaluation by calculating the Gini concordance, which involves the effective period and the amount of the data. Firstly, since the data is always mixed with some redundant or invalid parts, engineers need to process the data to capture the useful segments, seeking out the information which actually reflects the characteristic and uniqueness of the dynamic output. Secondly, according to (15) (19) in Sections 2.3 and 2.4, the output dynamic parameters must have the same amount of data; however, the output parameters are always in different sizes, suggesting that a new concept named effective period of output dynamic properties intended as a measure of deterministic of valid data sections should be proposed. Generally speaking, the effective period of output dynamic properties can be broadly categorized into three types. The graphical representation of these types is shown in Figure 5. Taking the groups of vectors in Figure 5 as an example, three vectors of the dynamic output are expressed as follows:

$$
\begin{aligned}
& \Theta_{A}=\left(\Theta_{A 1}, \Theta_{A 2}, \ldots, \Theta_{A n_{A}}\right), \\
& \Theta_{B}=\left(\Theta_{B 1}, \Theta_{B 2}, \ldots, \Theta_{B n_{B}}\right), \\
& \Theta_{C}=\left(\Theta_{C 1}, \Theta_{C 2}, \ldots, \Theta_{C n_{C}}\right) .
\end{aligned}
$$

The following part of this section gives the definition of the effective period and illustrates the approach of unifying the amount of data.

(1) Dynamic Output of Type I. The first is the stable output without the trend toward convergence or the cutoff points. As shown in Figure 5(a), curves I and II represent the dynamic output, and the two parameters do not converge to a constant and have no cutoff points. In this situation, the effective period $t_{E}$ is defined as the same time points

$$
t_{E}=t_{A}=t_{B}=t_{C}
$$

The valid data of this type is shown in Figure 5(a) as the time interval $\left[0, t_{A B C}\right]$ and the amount of data is unified naturally. So we have the final dynamic output vectors

$$
\begin{aligned}
& \Theta_{A}^{N}=\Theta_{A}=\left(\Theta_{A 1}, \Theta_{A 2}, \ldots, \Theta_{A n_{A}}\right), \\
& \Theta_{B}^{N}=\Theta_{B}=\left(\Theta_{B 1}, \Theta_{B 2}, \ldots, \Theta_{B n_{B}}\right), \\
& \Theta_{C}^{N}=\Theta_{C}=\left(\Theta_{C 1}, \Theta_{C 2}, \ldots, \Theta_{C n_{C}}\right),
\end{aligned}
$$

which satisfy

$$
n_{A}=n_{B}=n_{C} .
$$

(2) Dynamic Output of Type II. The second is that the output (angular) displacement is converged to a value but the durations to convergence points are not the same as each other. The concept is illustrated in Figure 5(c) and curves I, II, and III converge to the constant $\theta_{t}$ at the time points of $t_{A}$, $t_{B}$, and $t_{C}$. The effective periods of these output parameters are formulated as $t_{A}, t_{B}$, and $t_{C}$, respectively. Namely,

$$
t_{E}=t_{A}, t_{B}, t_{C} \text {. }
$$

The amount of data will be diverse if the same time interval is selected. In order to adjust the $\Theta-t$ data into the same amount, the effective period is evaluated by the $\dot{\Theta}-\Theta$ curves. At last, according to (20) we obtain the output dynamic vector as

$$
\begin{aligned}
& \Theta_{A}=\left(\Theta_{A 1}, \Theta_{A 2}, \ldots, \Theta_{A n_{A}}\right), \\
& \Theta_{B}=\left(\Theta_{B 1}, \Theta_{B 2}, \ldots, \Theta_{B n_{B}}\right), \\
& \Theta_{C}=\left(\Theta_{C 1}, \Theta_{C 2}, \ldots, \Theta_{C n_{C}}\right), \\
& \dot{\Theta}_{A}=\left(\dot{\Theta}_{A 1}, \dot{\Theta}_{A 2}, \ldots, \dot{\Theta}_{A n_{A}}\right), \\
& \dot{\Theta}_{B}=\left(\dot{\Theta}_{B 1}, \dot{\Theta}_{B 2}, \ldots, \dot{\Theta}_{B n_{B}}\right), \\
& \dot{\Theta}_{C}=\left(\dot{\Theta}_{C 1}, \dot{\Theta}_{C 2}, \ldots, \dot{\Theta}_{C n_{C}}\right) .
\end{aligned}
$$

By Lagrange's interpolation [30], the vectors $\dot{\Theta}_{B}$ and $\dot{\Theta}_{C}$ can be described by the $m$-order polynomial

$$
\begin{gathered}
\varphi_{B m}(x)=b_{0}+b_{1} x+b_{2} x^{2} \cdots b_{m} x^{m}, \\
\varphi_{C m}(x)=c_{0}+c_{1} x+c_{2} x^{2} \cdots c_{m} x^{m}
\end{gathered}
$$




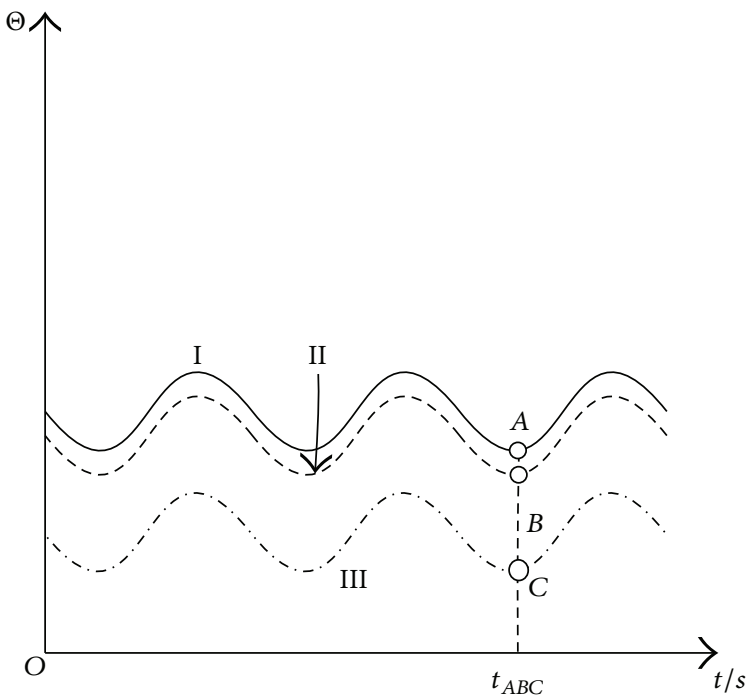

(a) Type I

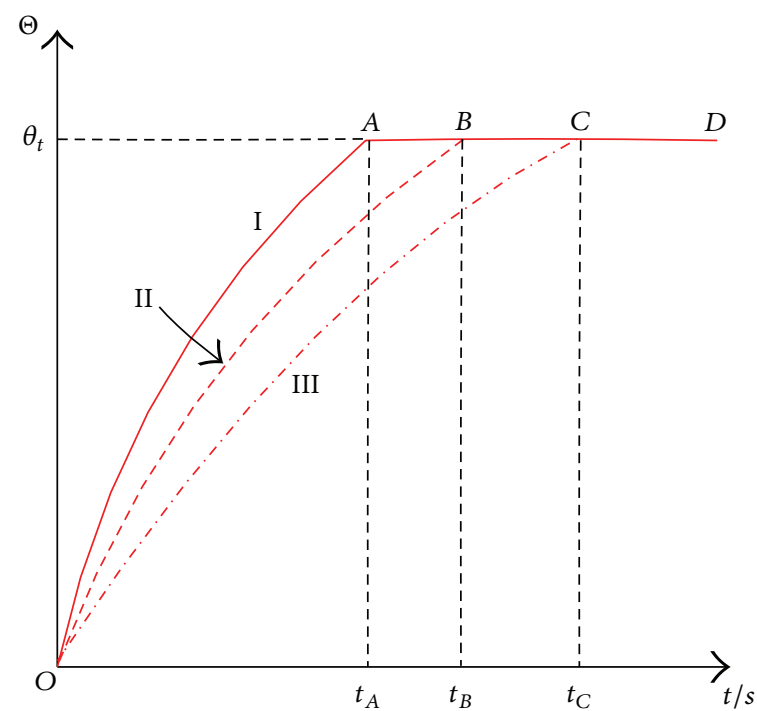

(b) Type II

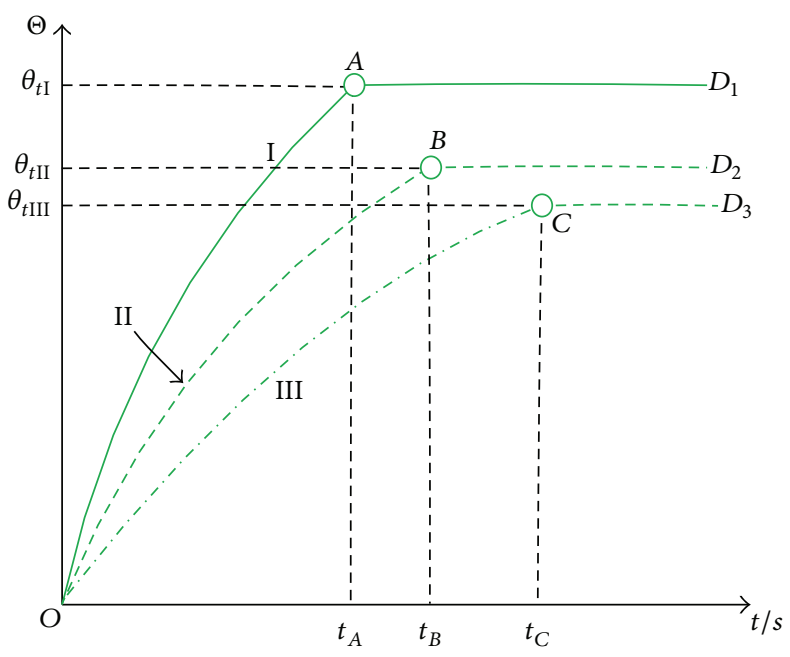

(c) Type III

FIGURE 5: Three types of output dynamic behavior.

which satisfies

$$
\begin{aligned}
& \varphi_{B m}\left(\Theta_{B j}\right)=\dot{\Theta}_{B j}, \\
& \varphi_{C m}\left(\Theta_{C j}\right)=\dot{\Theta}_{C j} .
\end{aligned}
$$

So the final dynamic output can be calculated by

$$
\begin{gathered}
\dot{\Theta}_{A}^{N}=\dot{\Theta}_{A}, \\
\dot{\Theta}_{B}^{N}=\left\{\dot{\Theta}_{B i}^{N}\right\}, \quad \dot{\Theta}_{B i}^{N}=\varphi_{B m}\left(\Theta_{A i}\right), \\
\dot{\Theta}_{C}^{N}=\left\{\dot{\Theta}_{C i}^{N}\right\}, \quad \dot{\Theta}_{C i}^{N}=\varphi_{C m}\left(\Theta_{A i}\right), \\
i=1,2, \ldots, n_{A}
\end{gathered}
$$

which satisfies the condition that the dimensions of the vectors are the same.

(3) Dynamic Output of Type III. The third type is that the output (angular) displacements are converged to different values and the duration to convergence points are not the same as each other. The concept is illustrated in Figure 5(c) and curves I, II, and III converge to the constants $\theta_{t \mathrm{I}}, \theta_{t \mathrm{II}}$, and $\theta_{t I I I}$ at the time points of $t_{A}, t_{B}$, and $t_{C}$. The effective period of these output parameters is formulated as $t_{A}, t_{B}$, and $t_{C}$, respectively. In order to ensure the unity of the amount of data, two ways of data transformation are proposed in this paper. The first is the data selection technique and the second is the data scaling technique.

(a) Data Selection. Data selection employs the method of selecting the numerical points uniformly to keep the amount 
of data the same as each other. Take Figure 5(c) as an example; the sizes of the vectors satisfy

$$
n_{A}<n_{B}<n_{C}
$$

The final output dynamic vectors can be written as

$$
\begin{aligned}
& \Theta_{A}^{N}=\Theta_{A}, \\
& \Theta_{B}^{N}=\left\{\Theta_{B k}^{N}\right\}_{k=k(i), i=1,2, \ldots, n_{A}} \\
& \Theta_{C}^{N}=\left\{\Theta_{C k}^{N}\right\}_{k=k(i), i=1,2, \ldots, n_{A}},
\end{aligned}
$$

where

$$
k=1+\left\lfloor\frac{n_{B}}{n_{A}}\right\rfloor(i-1) .
$$

And $\left\lfloor n_{B} / n_{A}\right\rfloor$ denotes the integer part of $\left(n_{B} / n_{A}\right)$.

(b) Data Scaling. The second way to adjust data amount is using the data scaling method. The normalization form of (23) is stated as

$$
\begin{aligned}
& \widehat{\Theta}_{A}=\frac{\Theta_{A}}{\max \left\{\Theta_{A i}\right\}}, \\
& \widehat{\Theta}_{B}=\frac{\Theta_{B}}{\max \left\{\Theta_{B i}\right\}}, \\
& \widehat{\Theta}_{C}=\frac{\Theta_{C}}{\max \left\{\Theta_{C i}\right\}}, \\
& \left(i=1,2, \ldots, n_{A}\right) .
\end{aligned}
$$

Consulting the approach for Type II, the (angular) velocity of $\dot{\widehat{\Theta}}_{A}, \dot{\widehat{\Theta}}_{B}$, and $\dot{\widehat{\Theta}}_{C}$ can also be computed by the differential method. Then, the curves of Type III are transformed into the ones with the same feature as the one of Type II shown in Figure 6(b). Consequently, the valid data of Type III for Gini concordance can be expressed by the curves $\dot{\widehat{\Theta}}_{A}-\widehat{\Theta}_{A}$, as shown in Figure 6(d). Then, the final dynamic output vectors $\dot{\hat{\Theta}}_{A}^{N}, \dot{\widehat{\Theta}}_{B}^{N}$, and $\dot{\widehat{\Theta}}_{C}^{N}$ can be obtained by the approach as that of Type II.

2.5. Severity Ranking by Gini Concordance. In this paper, the main conception of fault severity evaluation is processed by comparing the dynamic output of the ideal system with faulty system. Aiming at getting the severity ranking, the sample version of Gini concordance is discussed. The normal one is defined as the ideal output, and the Gini concordance measure which lies in the interval $[0,1]$ is used to evaluate the concordance between the ideal output and the abnormal output. If the Gini concordance is near to 1 , the output is as good as the normal output. Otherwise the output is far different from the normal state, indicating that the fault is much more serious. Take the dynamic output shown in Figure 7(a) as an example; curve $A$ represents the normal dynamic output (such as displacement, velocity, and acceleration), and curves
$B \sim E$ illustrate the dynamic output with four types of faults with the notations $B \sim E$. The Gini concordance shown in Figure $7(\mathrm{~b})$ indicates that the output of fault $D$ has the largest value, which means the output of this fault is the closet to the ideal one. Furthermore, it is quite evident that the fault $D$ is the least crucial among all the faults. In addition, the severity ranking of faults via Gini concordance can be summarized as $B>C>E>D$. Strategies for reliability design have the proneness that the faults of $B$ and $C$ should be largely concentrated to propose concepts and concrete approaches for eliminating faults.

By (15), we define $\theta_{X}^{N}=\left(\theta_{X(1)}^{N}, \theta_{X(2)}^{N}, \ldots, \theta_{X\left(n_{A}\right)}^{N}\right)_{1 \times n_{A}}(X=$ $A, B, C)$ which denotes the ranks in a sample of size $n_{A}$ of three vectors $\Theta_{X}^{N}(X=A, B, C)$, respectively. Likewise the vectors $\dot{\theta}_{X}^{N}=\left(\dot{\theta}_{X(1)}^{N}, \dot{\theta}_{X(2)}^{N}, \ldots, \dot{\theta}_{X\left(n_{A}\right)}^{N}\right)_{1 \times n_{A}}(X=$ $A, B, C)$ represent the ranks in a sample of size $n$ of three vectors $\dot{\Theta}_{X}^{N}(X=A, B, C)$, respectively. The method of calculating the three types of output is described as follows.

(1) Dynamic Output of Type I. As shown in Figure 6(a), the output in the time interval $\left[0, t_{A B C}\right]$ is stable and has no value to convergence. The Gini concordance can be directly used for concordance measurement. The formulae of Gini concordance between curve I and curve II can be written as [21]

$$
\gamma=\frac{1}{\left\lfloor n_{A}^{2} / 2\right\rfloor}\left[\sum_{i=1}^{n}\left|\theta_{A i}^{N}+\theta_{B i}^{N}-n-1\right|-\sum_{i=1}^{n}\left|\theta_{A i}^{N}-\theta_{B i}^{N}\right|\right] .
$$

Then, the Gini concordance between curve II and curve III can be computed by

$$
\gamma=\frac{1}{\left\lfloor n_{A}^{2} / 2\right\rfloor}\left[\sum_{i=1}^{n}\left|\theta_{B i}^{N}+\theta_{C i}^{N}-n-1\right|-\sum_{i=1}^{n}\left|\theta_{B i}^{N}-\theta_{C i}^{N}\right|\right] .
$$

(2) Dynamic Output of Type II. The second type is that the output (angular) displacement is converged to a value but the duration to convergence points is different from each other. According to Section 2.4, the Gini concordance between curve I and curve II is [21]

$$
\gamma=\frac{1}{\left\lfloor n_{A}^{2} / 2\right\rfloor}\left[\sum_{i=1}^{n}\left|\dot{\theta}_{A i}^{N}+\dot{\theta}_{B i}^{N}-n-1\right|-\sum_{i=1}^{n}\left|\dot{\theta}_{A i}^{N}-\dot{\theta}_{B i}^{N}\right|\right] .
$$

And the Gini concordance between curve II and curve III is

$$
\gamma=\frac{1}{\left\lfloor n_{A}^{2} / 2\right\rfloor}\left[\sum_{i=1}^{n}\left|\dot{\theta}_{B i}^{N}+\dot{\theta}_{C i}^{N}-n-1\right|-\sum_{i=1}^{n}\left|\dot{\theta}_{B i}^{N}-\dot{\theta}_{C i}^{N}\right|\right] .
$$

(3) Dynamic Output of Type III. The third type is that the output (angular) displacement is converged to different values but the durations to convergence points are different from each other. The Gini concordance is researched in the following two aspects: data selection and data scaling. In the former, Gini concordance can be computed by (31) (32). In the latter, Gini concordance can be calculated by (33) (34). 


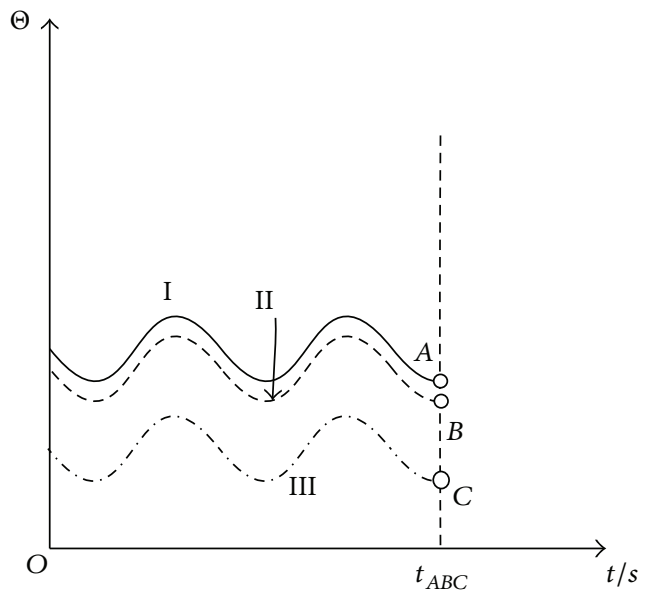

(a) Type I

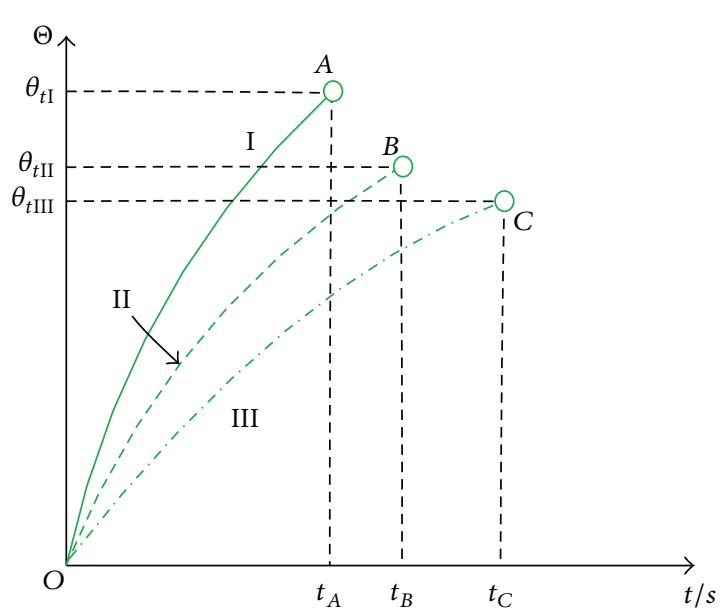

(c) Type III (data selection)

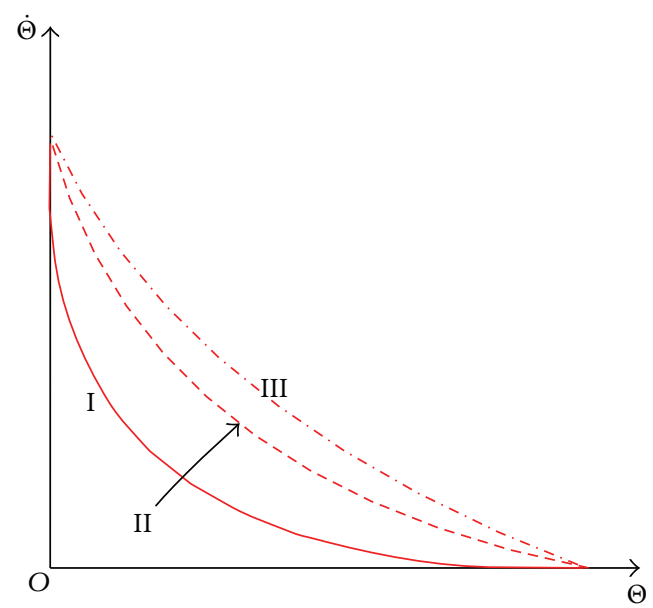

(b) Type II

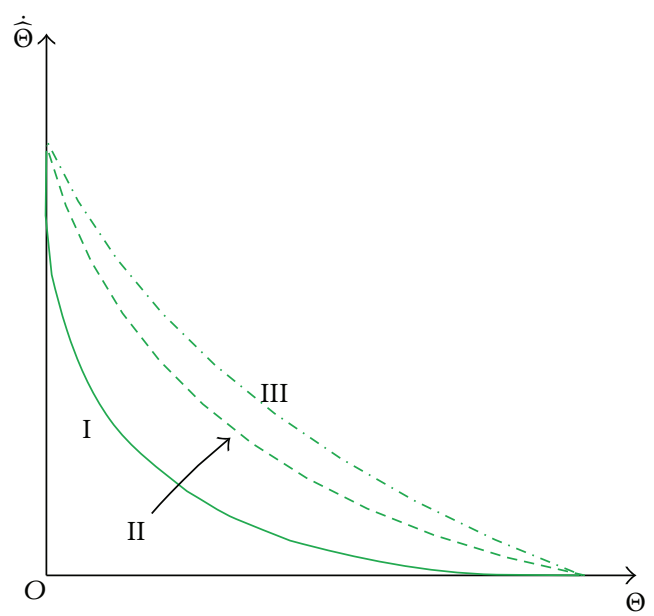

(d) Type III (data scaling)

FIGURE 6: Valid data for Gini concordance.

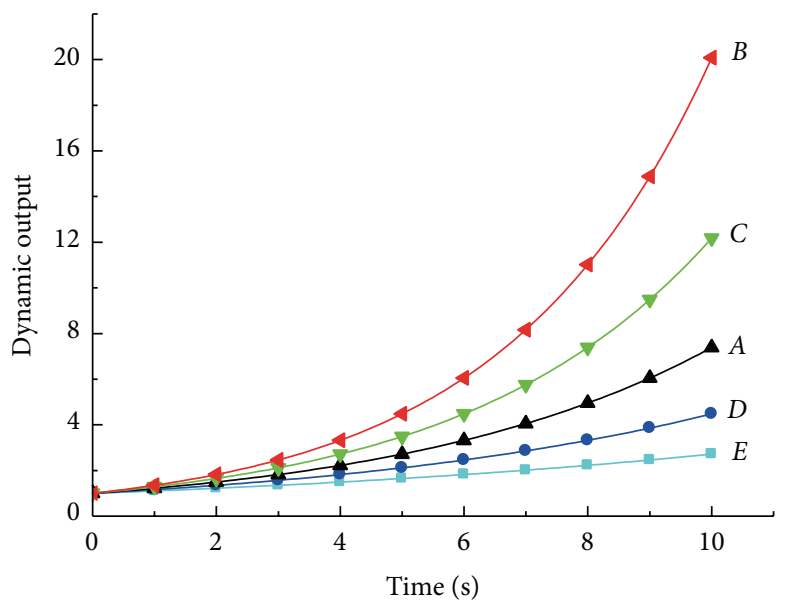

(a) Dynamic output

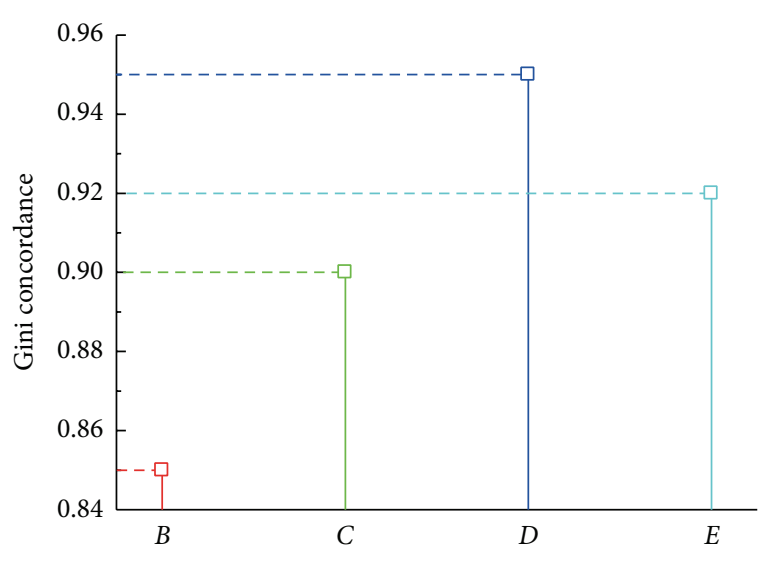

(b) Gini concordance

FIGURE 7: Principle of severity ranking by Gini concordance. 
TABLE 4: Design strategies for faults in common mechanical system.

\begin{tabular}{lll}
\hline Number & \multicolumn{1}{c}{ Faults } & Main design strategies \\
\hline 1 & Wear & (1) Change material of the friction pair; (2) add high-performance lubricant; (3) optimize the load. \\
2 & Crack and fracture & (1) Make the structure stronger; (2) optimize the structure to minimize the stress \\
3 & Deadlocking of joints/slider & $\begin{array}{l}\text { (1) Change the material of the friction pair; (2) optimize the protective structure; (3) improve the } \\
\text { lubrication }\end{array}$ \\
4 & Lack of energy & (1) Using redundancies as backups; (2) change the motor with higher power \\
5 & Slip of the belt & (1) Change the material of the friction pair; (2) improve the preload in the belt \\
6 & The others & Develop strategies depending on the circumstances
\end{tabular}

2.6. Fault Severity Evaluation and Improvement Strategies. The ranking of the Gini concordance reveals the severity of different faults which helps the engineers detect the fatal fault embedded in the system. It is evident that the more serious anomaly owns the larger value of Gini concordance. Therefore, these types of fault require special attention. The design strategies for typical faults in the mechanical system can be categorized as the wear, crack/fracture, deadlocking of joints, lack of energy, and the others. Generally speaking, the main improvement strategies in design stage for faults in common mechanical systems are shown in Table 4.

\section{Case Study}

3.1. Fault Mode of the Solar Array Mechanism. Solar arrays are one of the most vital links to spacecraft mission success because providing reliable power over the anticipated mission life is critical to spacecraft $[1,30]$. The entire running process includes three stages; that is, the deployable solar array is first folded and then deployed and locked in the orbit and finally oriented to the sun to generate power for spacecraft. The running process of a typical deployable solar array is shown in Figure 8. The catastrophic faults occur frequently in mechanical system of the solar array, which lead to the fault of the entire spacecraft and cause enormous economic loss [1]. Therefore, it is meaningful to study the characteristics of the fault mechanism and provide effective ways to improve its reliability.

In general, the solar array mechanism consists of three kinds of mechanisms $[15,27,31]$, that is, the hold-down and release mechanism, the deployable-locking mechanism, and the orientation mechanism. Take the solar array of DFH3 satellite which was launched in 1997 as an example; the hold-down and release mechanism contains seven explosive bolts that must be cut off by the knives after the instruction of deployment transmitted from the satellite in orbit. The structure of the hold-down and release mechanism is graphically sketched in Figure 9(a). As shown in Figure 9(b), the solar array is composed of four panels marked with $P_{1}, P_{2}, P_{3}$, and $P_{4}$. In addition, the deployable mechanism is the hinge, consisting of the driving spring, locking spring, closed cable loop (CCL), and pins with clearances. The hinges are marked from the satellite to the outside of the solar array as $H_{1}, H_{2}, H_{3}, H_{4}$. Torsion springs are often chosen to drive the solar array and the locking spring is used to make the solar array fixed after deployment. The driving mechanism
TABLE 5: Markers and events of FTA.

\begin{tabular}{ll}
\hline Marker (FTA) & Event \\
\hline$T$ & Fault of the solar array system \\
$B$ & Fault during the release of the solar array \\
$C$ & Faults during deployment process \\
$D$ & Faults during locking process \\
$E$ & Fault of orientation to the sun \\
$B_{1}$ & Other faults of mechanical system \\
$B_{2}$ & Deadlocking in hinges \\
$B_{3}$ & Fault of the driving torsion spring \\
$E_{3}$ & Fault of the CCL \\
$X_{1}$ & Vibration of panels induced by thermal \\
$X_{2}$ & deformation \\
$X_{3}$ & Electronic arcing is out of service \\
$X_{8}$ & Fault of the cutters \\
$X_{5}$ & Harsh thermal environment in space \\
$X_{6}$ & Fault of the grease used in hinges between panels \\
$X_{7}$ & Insufficient torque of the main torsion spring \\
$X_{8}$ & Insufficient torque of the reserved torsion spring \\
$X_{9}$ & Insufficient preload of the cable \\
$X_{10}$ & Poor thermal characteristic of the cable \\
$X_{11}$ & Inappropriate driving torque of the locking \\
$X_{12}$ & torsion spring \\
$X_{13}$ & Fault of the motor \\
$X_{14}$ & Fault of the transmission unit \\
\hline & Impact caused by particles in space \\
& mad thermal characteristic of honeycomb \\
&
\end{tabular}

in the hinges $H_{1} \sim H_{4}$ are noted as $D_{1} \sim D_{4}$ and the locking mechanism in the hinges $H_{1} \sim H_{4}$ are noted as $L_{1} \sim L_{4}$. The CCLs meet the requirement of motion coordination and synchronization during deployment, which are organized as $\mathrm{CCL}_{1}, \mathrm{CCL}_{2}$, and $\mathrm{CCL}_{3}$ in Figure 9(c). The solar panels are oriented to the sun by orientation mechanism that is composed of the stepping motor and the harmonic reducer. In this paper, only the deployment of solar array is considered, so the target mechanism is comprised of the panels, the driving spring, locking spring, and the CCLs.

According to the method shown in Section 2.1, Table 5, and Figure 10 we summarize the markers and events of 


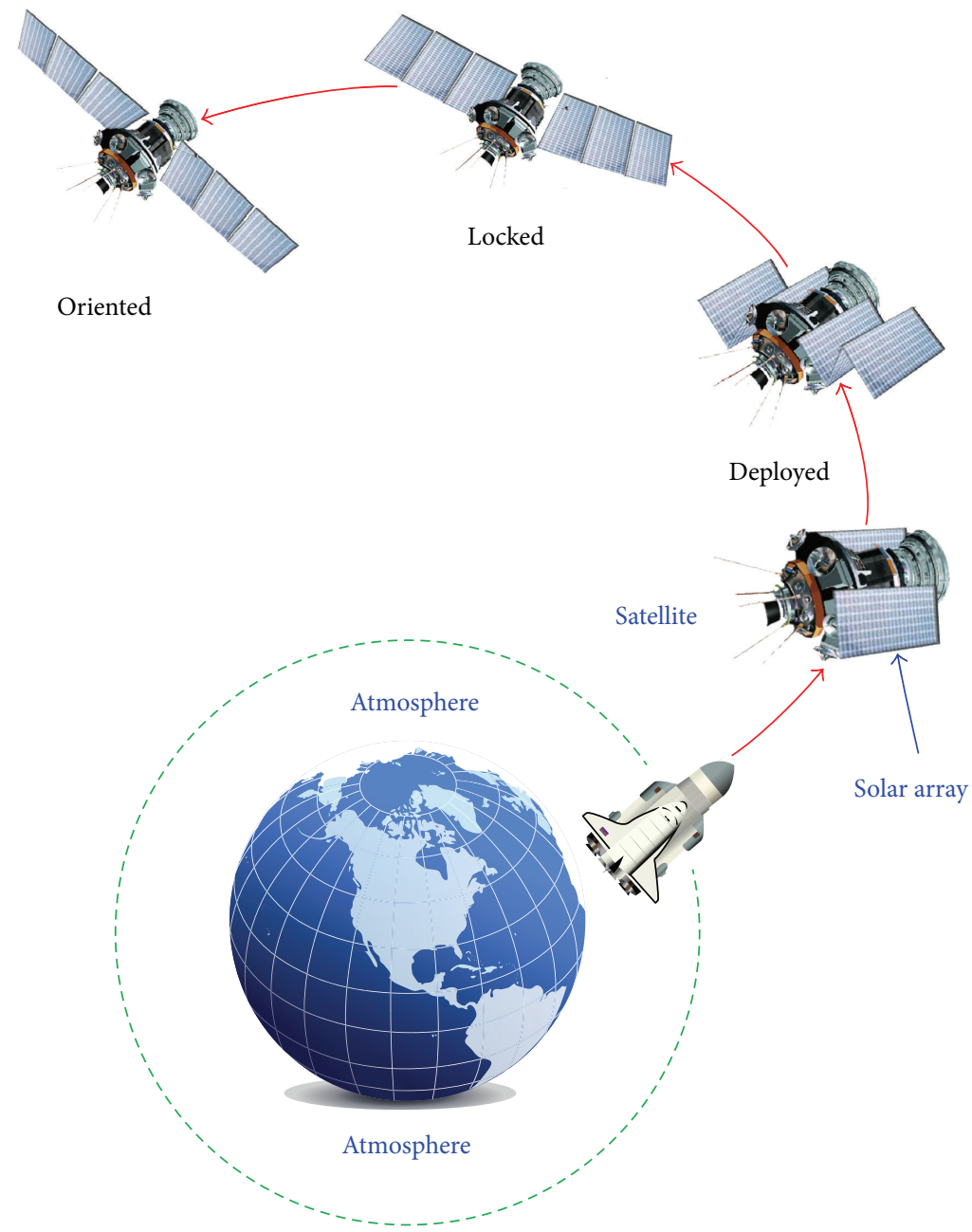

FIGURE 8: Operating principle of a deployable solar array.

the FTA model [29], in which $X_{i}(i=1,2, \ldots, 14)$ are the notations of the bottom events of the system. Only the course of deployment and locking is considered in this paper.

We invited four experts on the research field of spacecraft engineering or reliability analysis to get the scores of the bottom events and the history data are also used to acquire the adjustment parameter of the $i$ th bottom event, namely, $A_{i}(i=1,2, \ldots, n)\left(A_{i} \geq 1\right)$. According to the Delphi method, four kinds of most dangerous faults are selected. They are deadlocking of hinges, fault of the CCLs, fault of the locking mechanism in the hinges, and fault of the driving torsion springs. The following part of Section 3 will center attention on these four types of faults, exploring the ranks of the fault existed in the components of the related components.

3.2. Dynamical Modeling and Experimental Setup. The dynamical model and experimental setup are the tools for simulation-based technique and the execution-based technique, respectively. So the dynamic modeling and its experimental validation are investigated in the following parts of this section.
3.2.1. Dynamical Modeling of the Solar Array. Referring to Figure 9(c), the equations of motion of a multibody system are efficiently derived by multibody formalisms that are based on data which describe the system elements and topology. Here, the Lagrangian equations of type one are discussed. They are written as $n_{z}$ redundant variables of position and velocity $-n_{z} \times 1$-matrices $z_{\mathrm{I}}$ and $z_{\mathrm{II}}$-of the $n$ bodies and the constraint forces $-n_{c} \times 1$-matrix $\lambda$ of the $n_{G}$ joints. The differential-algebraic equations (DAE) of motion in the socalled descriptor form with the dimension DAE $=2 n_{z}+n_{c}$ are given as [15]

$$
\begin{aligned}
& \dot{z}_{\mathrm{I}}=Z z_{\mathrm{I}}, \\
& M \ddot{z}_{\mathrm{I}}-G^{T} \lambda=h_{a}, \\
& g=0 \quad \text { or } \quad G z_{\mathrm{II}}=\kappa, \\
& \quad \text { with } G=\frac{\partial g}{\partial z_{\mathrm{I}}},
\end{aligned}
$$

where $Z=Z\left(z_{\mathrm{I}}\right)$ is the matrix of kinematic equations, $M=$ $M\left(z_{\mathrm{I}}\right)$ is the mass matrix, $h_{a}=h_{a}\left(z_{\mathrm{I}}, z_{\mathrm{II}}, t\right)$ is the matrix of generalized applied forces due to stresses, gravity, body and 


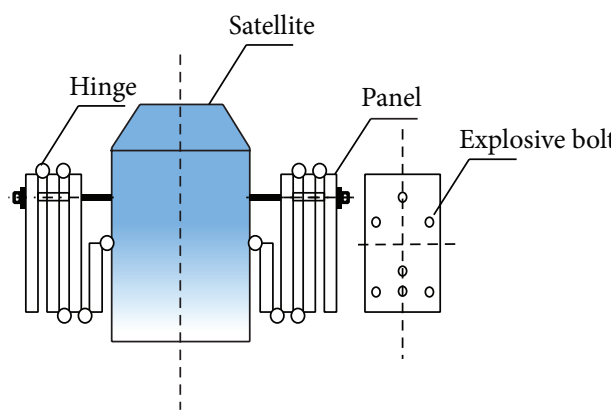

(a)

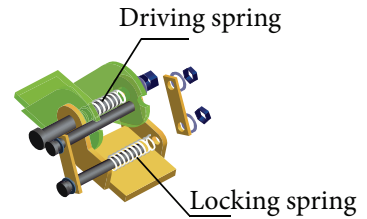

(b)

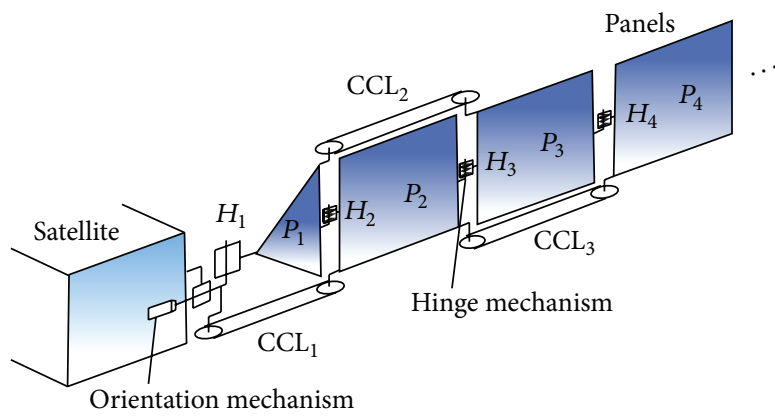

(c)

FIGURE 9: Mechanisms of the solar array.

surface forces, and torques, $g=g\left(z_{\mathrm{I}}, t\right)$ is the $n_{c} \times 1$-matrix of the implicit position constraint equations, and $G=G\left(z_{\mathrm{I}}, t\right)$ is the $n_{c} \times n_{z}$-constraint matrix, the Jacobian of $g$. Matrix $\kappa=$ $\kappa(t)$ defines functions of time at joints. The matrices in (1) are the sum of matrices that describe a single body and its forces and torques (index $i$ ) and a single joint (index $s$ ). Thus,

$$
\begin{gathered}
z_{\mathrm{I}}=\left[z_{\mathrm{I}}^{i}\right], \quad z_{\mathrm{II}}=\left[z_{\mathrm{II}}^{i}\right], \quad \lambda=\left[\lambda^{S}\right], \quad g=\left[g^{S}\right], \\
Z=\operatorname{diag}\left[Z^{i}\right], \quad M=\operatorname{diag}\left[M^{i}\right], \quad h_{a}=\left[h_{a}^{i}\right] \\
n_{z}=\sum_{i=1}^{n} n_{z}^{i}, \quad n_{c}=\sum_{i=1}^{n_{G}} n_{c}^{S}, \\
i=1,2, \ldots, n, \quad s=1,2, \ldots, n_{G} .
\end{gathered}
$$

The theoretical analysis, such as (35), helps us to know which parameters should be considered and how to set them. On the basis of (35) (36), the deployment of the spacecraft solar array is simulated by the multibody program ADAMS (Figure 11). The model is comprised of three aluminum panels $\left(P_{2}, P_{3}\right.$, and $P_{4}$ as shown in Figure 9$)$ of $300 \mathrm{~mm} \times 200 \mathrm{~mm} \times$ $5 \mathrm{~mm}$ and one yoke in the size of $150 \mathrm{~mm} \times 200 \mathrm{~mm} \times 5 \mathrm{~mm}$ and the three CCLs are appended to the spacecraft. The driving springs and locking springs are installed in the hinges.

3.2.2. Setup of the Deployable Solar Array. The organization of the setup is organized identically to Figure 11, consisting of four panels, four hinges, three CCLs, and an orientation mechanism with a harmonic reducer. The sensors are

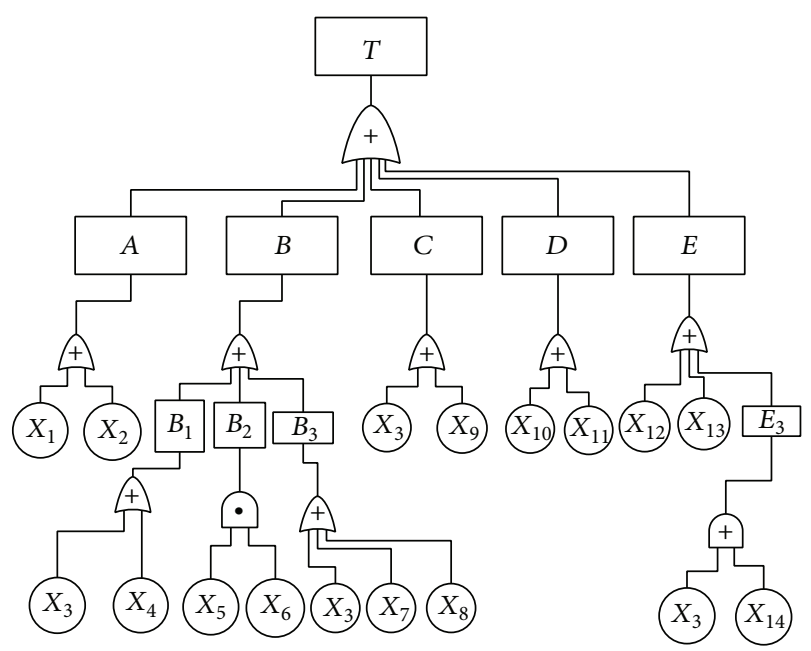

FIGURE 10: FTA model of the mechanical system of solar array.

installed in the hinges to measure the angular displacements. The schematic of the experiment system is shown in Figure 12. The signal collected from the angular displacement sensors is amplified and converted to the analog signal.

3.3. Fault Injection Technique for Solar Array Mechanism. Based upon the fault injection technique depicted in Section 2.2, all kinds of faults are injected into the solar array mechanism. Table 6 shows the simulation-based and execution-based fault injection techniques for the solar array mechanism, and the angular velocities in the hinges are 
TABLE 6: Main faults and the simulation method.

\begin{tabular}{|c|c|c|c|c|c|}
\hline Type & Fault & Sequence & $\begin{array}{l}\text { Simulation-based } \\
\text { technique } \\
\text { (parameter setting) } \\
\end{array}$ & $\begin{array}{l}\text { Execution-based } \\
\text { technique } \\
\text { (hardware setting) } \\
\end{array}$ & $\begin{array}{l}\text { Output angular displacement } \\
\text { in hinges }\end{array}$ \\
\hline \multirow{3}{*}{1} & \multirow{3}{*}{ Deadlocking of the hinges } & I & $\theta_{i}=0^{\circ} \quad(i=1,2,3,4)$ & $\begin{array}{l}\text { Insert the locking } \\
\text { pin in the hinge to } \\
\text { limit its rotation }\end{array}$ & $\begin{array}{c}\Theta^{S}=\left\{\Theta_{\mathrm{Ii}}^{S}\right\} \quad(i=1,2,3,4) \\
\Theta^{E}=\left\{\Theta_{\mathrm{I} i}^{E}\right\} \quad(i=1,2,3,4)\end{array}$ \\
\hline & & II & $\theta_{i}=10^{\circ} \quad(i=1,2,3,4)$ & $\begin{array}{l}\text { Insert the locking } \\
\text { pin in the hinge to } \\
\text { make it rotate only } \\
10 \text { degrees }\end{array}$ & $\begin{array}{c}\Theta^{E}=\left\{\Theta_{I I i}^{E}\right\} \quad(i=1,2,3,4) \\
\Theta^{S}=\left\{\Theta_{I I i}^{S}\right\} \quad(i=1,2,3,4)\end{array}$ \\
\hline & & III & $\theta_{i}=30^{\circ} \quad(i=1,2,3,4)$ & $\begin{array}{l}\text { Insert the locking } \\
\text { pin in the hinge to } \\
\text { make it rotate only } \\
30 \text { degrees }\end{array}$ & $\begin{array}{c}\Theta^{E}=\left\{\Theta_{\mathrm{IIIi}}^{E}\right\} \quad(i=1,2,3,4) \\
\Theta^{S}=\left\{\Theta_{\mathrm{IIIi}}^{S}\right\} \\
(i=1,2,3,4)\end{array}$ \\
\hline \multirow{3}{*}{2} & \multirow{3}{*}{ Fault of the CCLs } & III & $\mathrm{CCL}_{1}: F_{1}=F_{2}=0$ & Remove $\mathrm{CCL}_{1}$ & $\begin{array}{c}\Theta^{S}=\left\{\Theta_{\mathrm{IIIi}}^{S}\right\} \quad(i=1,2,3,4) \\
\Theta^{E}=\left\{\Theta_{\mathrm{IIIi}}^{E}\right\} \\
(i=1,2,3,4)\end{array}$ \\
\hline & & IV & $\mathrm{CCL}_{2}: F_{1}=F_{2}=0$ & Remove $\mathrm{CCL}_{2}$ & $\begin{array}{c}\Theta^{S}=\left\{\Theta_{\mathrm{IVi}}^{S}\right\} \quad(i=1,2,3,4) \\
\Theta^{E}=\left\{\Theta_{\mathrm{IV} i}^{E}\right\} \\
(i=1,2,3,4)\end{array}$ \\
\hline & & $\mathrm{V}$ & $\mathrm{CCL}_{3}: F_{1}=F_{2}=0$ & Remove $\mathrm{CCL}_{3}$ & $\begin{array}{c}\Theta^{S}=\left\{\Theta_{\mathrm{V} V}^{S}\right\} \quad(i=1,2,3,4) \\
\Theta^{E}=\left\{\Theta_{\mathrm{V} i}^{E}\right\} \quad(i=1,2,3,4)\end{array}$ \\
\hline \multirow{4}{*}{3} & \multirow{4}{*}{ Fault of the locking mechanism in the hinges } & VI & $\begin{array}{r}\text { Stiffness of locking } \\
\text { spring in } H_{1}: K_{T 1}=0\end{array}$ & $\begin{array}{l}\text { Remove the } \\
\text { locking torsion } \\
\text { spring in } H_{1}\end{array}$ & $\begin{array}{c}\Theta^{S}=\left\{\Theta_{\mathrm{VII} i}^{S}\right\} \quad(i=1,2,3,4) \\
\Theta^{E}=\left\{\Theta_{\mathrm{VIIi}}^{E}\right\} \\
(i=1,2,3,4)\end{array}$ \\
\hline & & VII & $\begin{array}{r}\text { Stiffness of locking } \\
\text { spring in } H_{2}: K_{T 1}=0\end{array}$ & $\begin{array}{l}\text { Remove the } \\
\text { locking torsion } \\
\text { spring in } \mathrm{H}_{2}\end{array}$ & $\begin{aligned} \Theta^{S}=\left\{\Theta_{\mathrm{VIII} i}^{S}\right\} \quad(i=1,2,3,4) & \Theta^{E}=\left\{\Theta_{\mathrm{VIII} i}^{E}\right\} \\
& (i=1,2,3,4)\end{aligned}$ \\
\hline & & VIII & $\begin{array}{r}\text { Stiffness of locking } \\
\text { spring in } H_{3}: K_{T 3}=0\end{array}$ & $\begin{array}{l}\text { Remove the } \\
\text { locking torsion } \\
\text { spring in } H_{3}\end{array}$ & $\begin{array}{c}\Theta^{S}=\left\{\Theta_{\mathrm{IX} i}^{S}\right\} \quad(i=1,2,3,4) \\
\Theta^{E}=\left\{\Theta_{\mathrm{IX} i}^{E}\right\} \\
(i=1,2,3,4)\end{array}$ \\
\hline & & IX & $\begin{array}{r}\text { Stiffness of locking } \\
\text { spring in } H_{4}: K_{T 4}=0\end{array}$ & $\begin{array}{l}\text { Remove the } \\
\text { locking torsion } \\
\text { spring in } H_{4} \\
\end{array}$ & $\begin{array}{c}\Theta^{S}=\left\{\Theta_{\mathrm{Xi}}^{S}\right\} \quad(i=1,2,3,4) \\
\Theta^{E}=\left\{\Theta_{\mathrm{X} i}^{E}\right\} \quad(i=1,2,3,4)\end{array}$ \\
\hline \multirow{4}{*}{4} & \multirow{4}{*}{ Fault of the driving torsion springs } & $\mathrm{XI}$ & $\begin{array}{r}\text { Stiffness of driving } \\
\text { spring in } H_{1}: K_{T 1}=0\end{array}$ & $\begin{array}{l}\text { Remove the } \\
\text { driving torsion } \\
\text { spring in } H_{1}\end{array}$ & $\begin{array}{c}\Theta^{S}=\left\{\Theta_{\mathrm{X} I i}^{S}\right\} \quad(i=1,2,3,4) \\
\Theta^{E}=\left\{\Theta_{\mathrm{XI} i}^{E}\right\} \\
(i=1,2,3,4)\end{array}$ \\
\hline & & XII & $\begin{array}{r}\text { Stiffness of driving } \\
\text { spring in } H_{2}: K_{T 2}=0\end{array}$ & $\begin{array}{l}\text { Remove the } \\
\text { driving torsion } \\
\text { spring in } \mathrm{H}_{2}\end{array}$ & $\begin{array}{c}\Theta^{S}=\left\{\Theta_{\mathrm{XIIi}}^{S}\right\} \quad(i=1,2,3,4) \\
\Theta^{E}=\left\{\Theta_{\mathrm{XIIi}}^{E}\right\} \\
(i=1,2,3,4)\end{array}$ \\
\hline & & XIII & $\begin{array}{r}\text { Stiffness of driving } \\
\text { spring in } H_{3}: K_{T 3}=0\end{array}$ & $\begin{array}{l}\text { Remove the } \\
\text { driving torsion } \\
\text { spring in } H_{3}\end{array}$ & $\begin{array}{c}\Theta^{S}=\left\{\Theta_{\mathrm{XIII} i}^{S}\right\} \quad(i=1,2,3,4) \\
\Theta^{E}=\left\{\Theta_{\mathrm{XIIII}}^{E}\right\} \\
(i=1,2,3,4)\end{array}$ \\
\hline & & XIV & $\begin{array}{r}\text { Stiffness of driving } \\
\text { spring in } H_{4}: K_{T 4}=0\end{array}$ & $\begin{array}{l}\text { Remove the torsion } \\
\text { spring in } H_{4}\end{array}$ & $\begin{aligned} \Theta^{S}=\left\{\Theta_{\mathrm{XIV} i}^{S}\right\} \quad(i=1,2,3,4) \\
\Theta^{E}=\left\{\Theta_{\mathrm{XIV} i}^{E}\right\} \\
(i=1,2,3,4)\end{aligned}$ \\
\hline
\end{tabular}

considered as the dynamic output which reflects the dynamic behavior of the solar array mechanism in deployment. $\Theta^{S}$ and $\Theta^{E}$ represent the simulation-based and the executionbased output of the angular displacements, respectively. Take the first type of fault as an example; the simulation-based technique and the execution-based technique are shown in the 4 th and 5 th columns of the table. If we want to simulate the deadlocking of the hinges, we could set $\theta_{i}=0^{\circ}(i=$ $1,2,3,4)$ as the simulation-based technique and insert the locking pin in the hinge to limit its rotation as the executionbased technique.

By solving the dynamic equations and conducting the experiments of the solar array mechanism with faults, the dynamic output can be acquired. The maximum relative error 
TABLE 7: $\varepsilon_{m}$ of the output between the simulation and experiments.

\begin{tabular}{|c|c|c|c|c|c|c|c|c|c|c|c|c|c|c|}
\hline$m$ & I & II & III & IV & $\mathrm{V}$ & VI & VII & VIII & IX & $\mathrm{X}$ & $\mathrm{XI}$ & XII & XIII & XIV \\
\hline Relative errors (\%) & 2.1 & 1.9 & 4.9 & 4.5 & 4.3 & 4.1 & 3.5 & 3.5 & 2.5 & 2.2 & 2.9 & 2.8 & 3.8 & 3.0 \\
\hline
\end{tabular}

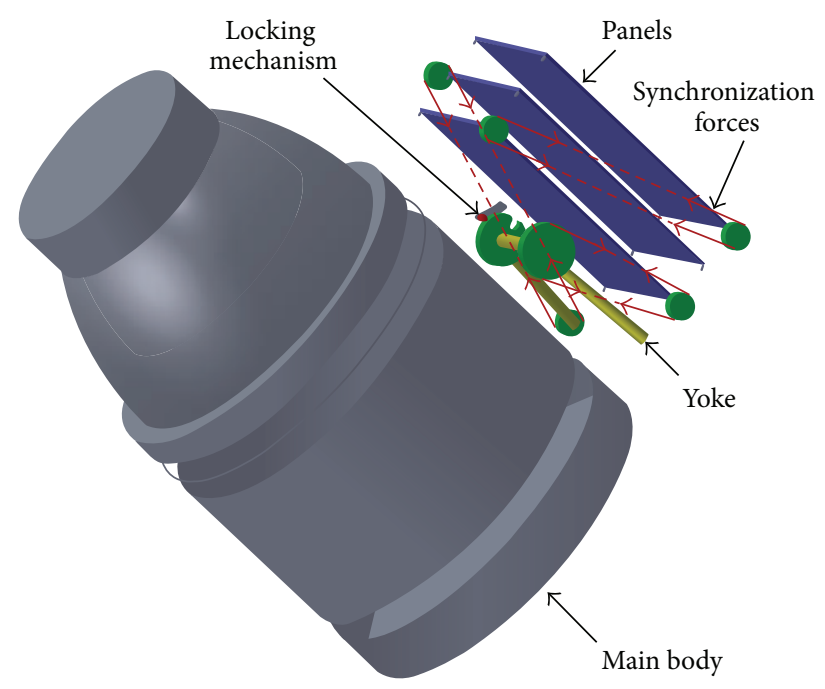

FIgURE 11: Solar array model in ADAMS.

which verifies the correctness and accuracy of the simulation is expressed as

$$
\begin{array}{r}
\varepsilon_{m i}=\max \left\{\left|\frac{\Theta_{m i q}^{S}-\Theta_{m i q}^{E}}{\Theta_{m i q}^{S}}\right|\right\}, \quad m=\mathrm{I}, \mathrm{II}, \ldots, \mathrm{XIV}, \\
i=1,2,3,4, \quad q=1,2, \ldots, w,
\end{array}
$$

where $\varepsilon_{m i}$ represents the relative error between $\Theta_{m i}^{S}$ and $\Theta_{m i}^{E}$ in the sequence $m$ and the vectors $\Theta_{m i}^{S}$ and $\Theta_{m i}^{E}$ are written as

$$
\begin{aligned}
& \Theta_{m i}^{S}=\left\{\Theta_{m i q}^{S}\right\}_{q=1,2, \ldots, w}, \\
& \Theta_{m i}^{E}=\left\{\Theta_{m i q}^{E}\right\}_{q=1,2, \ldots, w},
\end{aligned}
$$

in which $w$ is the number of the time points. Finally, the maximum relative error of the $m$ th sequence can be defined as

$$
\varepsilon_{m}=\frac{\left(\sum_{i=1}^{4} \varepsilon_{m i}\right)}{4}
$$

It should be noteworthy that every experiment is conducted for 3 times to get stable and convincing results. Table 7 represents the maximum relative errors between the dynamic output from the simulation and the experiments. It can be observed that the relative errors of the output between the simulation and experiments are all confined within $5 \%$, which proves the validity of the results generated from the simulation by using the fault injection technique. Therefore, in the following part, just the dynamic output from the simulation-based technique is used to accomplish the

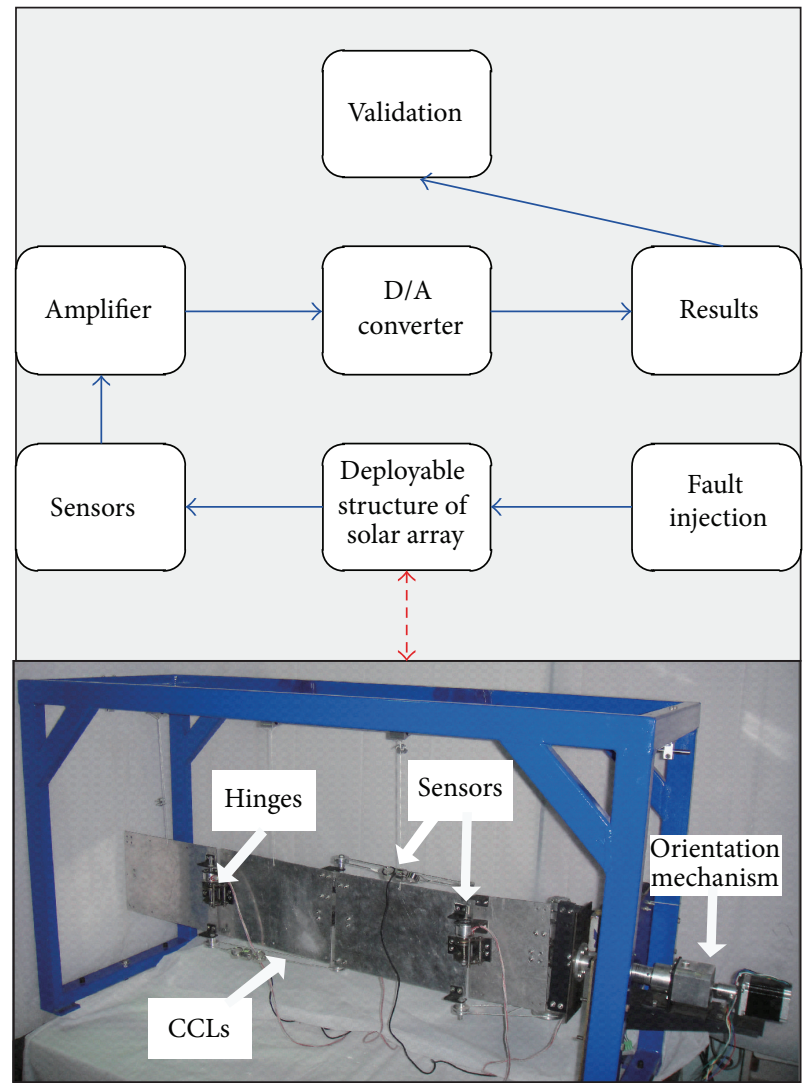

FIGURE 12: Experimental setup of the solar array mechanism.

reliability analysis of the subsystems via the Gini concordance measure.

3.4. Dynamic Behavior of the Solar Array Mechanism. Figure 13 shows the dynamic output of the normal and faulty deployment and the output angular displacements are paid close attention to measure the reliability of the system. Four types of the dynamic output of the angular displacements are shown as the typical examples of the dynamic output. As shown in Figure 13(a), the dynamic output of the normal deployment is treated as the ideal output, and the angular displacements of the four joints with different kinds of faults are shown in Figures 13(b) 13(f).

3.5. Gini Concordance Measure. Following the method introduced in Sections 2.3 2.5, the Gini concordance by comparing the ideal dynamic output with the output accompanied with faults can be calculated. As depicted in Table 6, four types of faults are considered in this paper. Figure 14 shows the average Gini concordance of the faults. The Gini concordances of the four types of faults are 0.4601, 0.8035, 


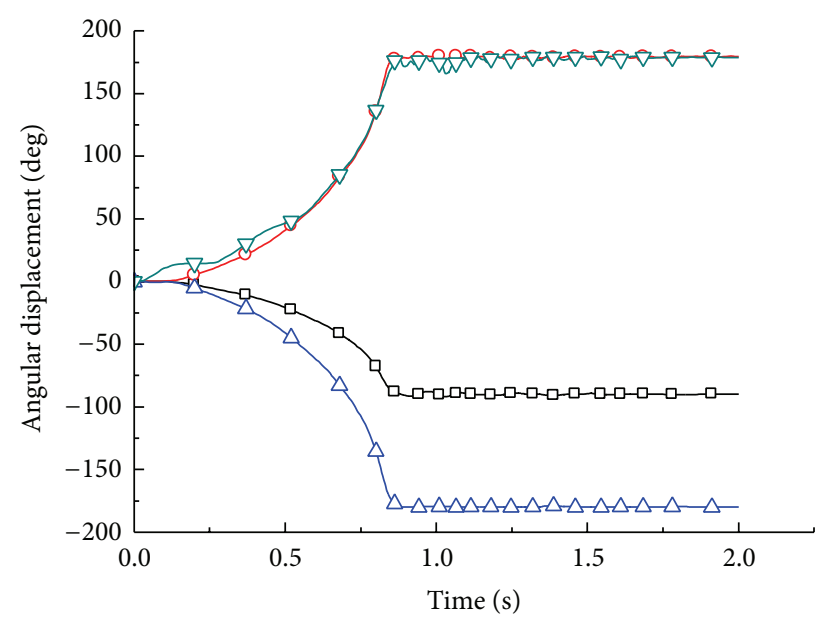

(a) Normal deployment

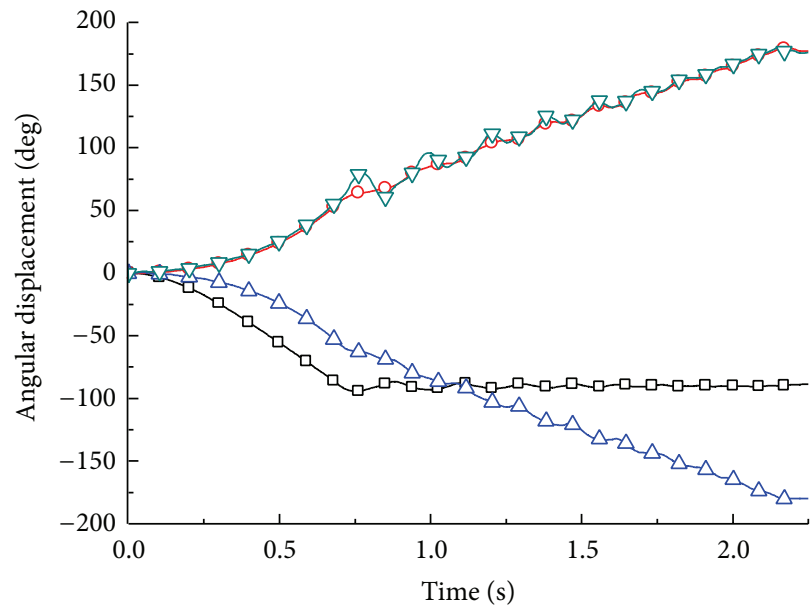

(c) Fault of $\mathrm{CCL}_{1}$

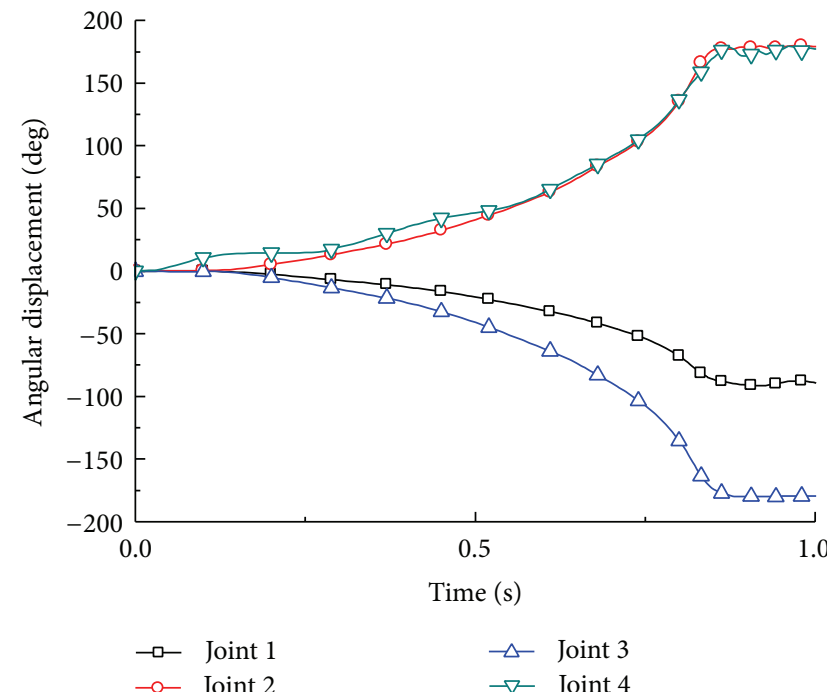

(e) Fault of the locking mechanism in $H_{1}$

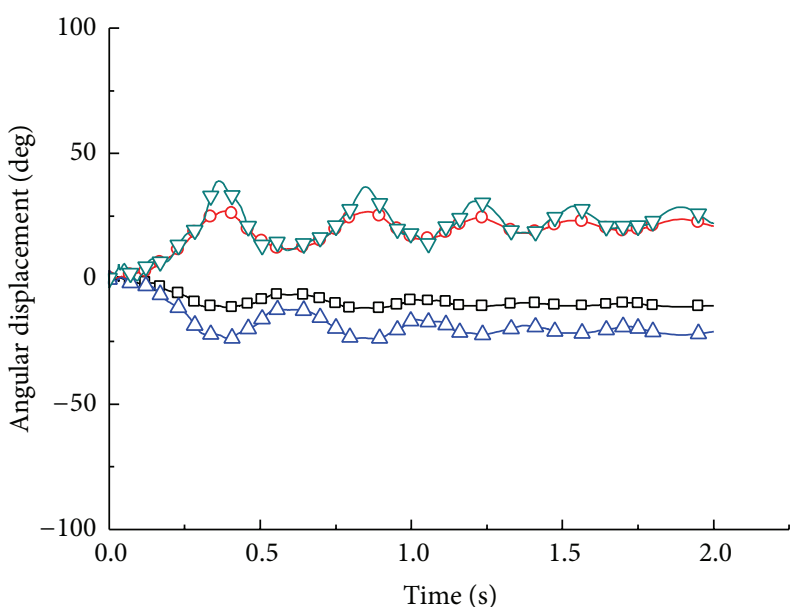

(b) Deadlocking of $H_{1}$

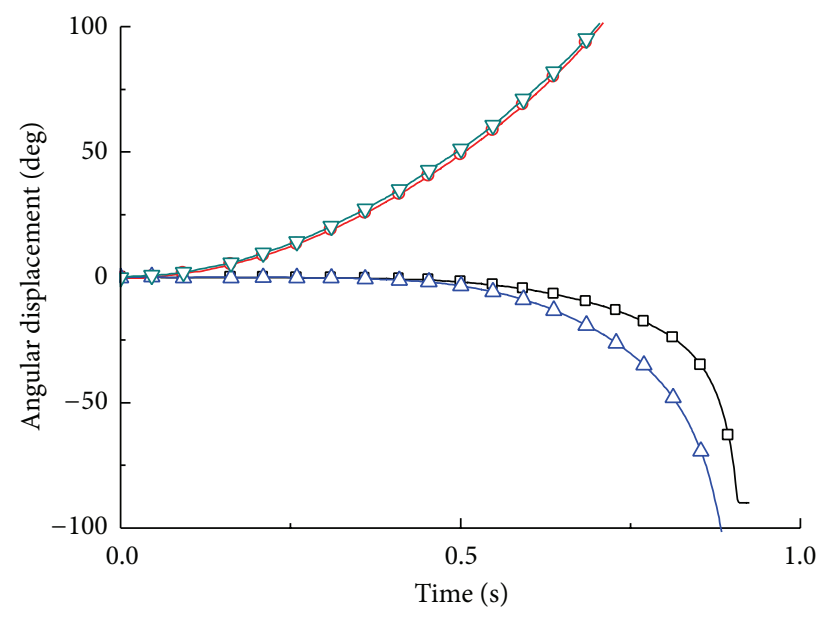

(d) Fault of $\mathrm{CCL}_{2}$

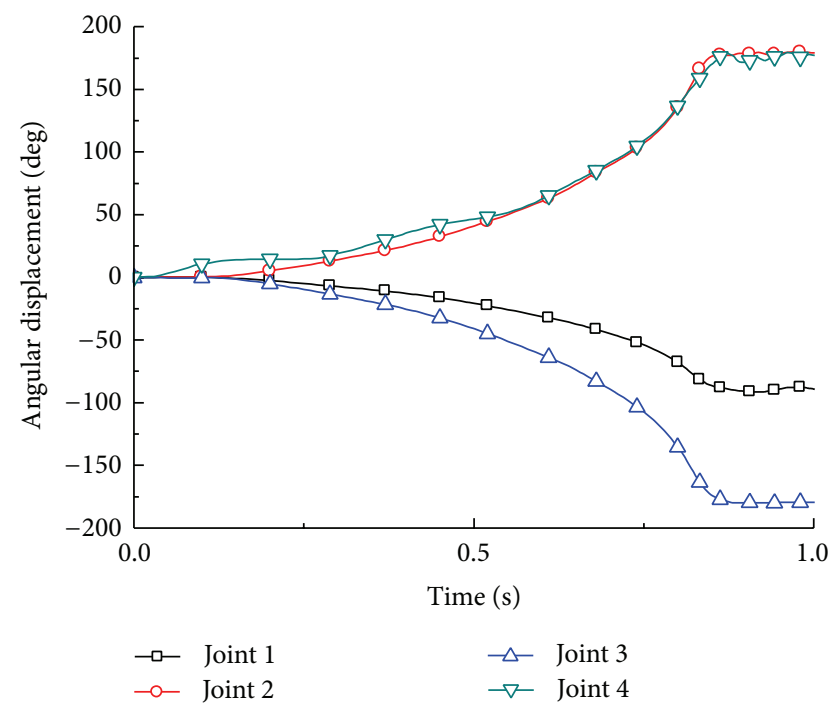

(f) Fault of the driving torsion springs in $H_{1}$

FIGURE 13: The dynamic output of the normal and faulty deployment. 


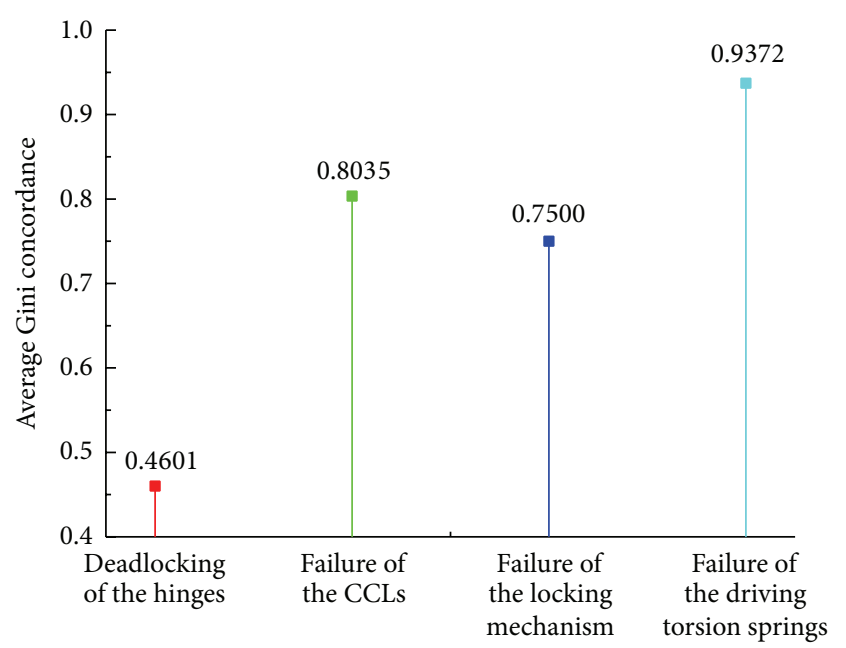

FIGURE 14: Average Gini concordance of the faults.

0.7500 , and 0.9372 , respectively, which shows the severity ranking of these faults that the deadlocking of the hinges is the most serious and the fault of the driving torsion spring is the slightest anomaly.

Table 8 shows the Gini concordance between the deployment with anomalies and the normal deployment. From the values in this table, the conclusion can be drawn as follows.

(1) The deadlocking in hinges is stuck with the deployment angle of $0^{\circ}, 10^{\circ}$, and $60^{\circ}$ with the notation deadlocking 1 , deadlocking 2 , and deadlocking 3 , respectively. The average values of the Gini concordance are $0,0.4841$, and 0.8962 , which indicates that the deadlocking is more serious in the initial stage of deployment of the solar array.

(2) As shown in Table 8, according to the average value of the Gini concordance, the fault of $\mathrm{CCL}_{2}$ has the greatest Gini concordance value, whereas the fault of $\mathrm{CCL}_{1}$ gets the smallest Gini concordance value. The severity ranking of this type of faults is that $\mathrm{CCL}_{1}>$ $\mathrm{CCL}_{3}>\mathrm{CCL}_{2}$, which means that the fault of the $\mathrm{CCL}_{1}$ is the most dangerous and the designers should pay more attention to this component of the system.

(3) The Gini concordance with the fault of the locking mechanism is summarized in Table 8 . The severity ranking is $L_{3}>L_{2}>L_{4}>L_{1}$ which reveals that the anomaly of locking mechanism $L_{3}$ is the most dangerous and needs to be especially reinforced.

(4) The faults of the driving torsion springs are not as serious as the other three kinds of faults (Figure 14, Table 8). Fault of the driving torsion spring $D_{1}$ is the weak link among all of the driving springs. As a result, the redundant backup or higher preload should be considered in reliability design.

After the statement of the basic results, details on the reliability design methods are discussed in later sections.
TABLE 8: Gini concordance of the dynamic output.

\begin{tabular}{lccccc}
\hline Fault & $H_{1}$ & $H_{2}$ & $H_{3}$ & $H_{4}$ & Average \\
\hline Deadlocking 1 & 0 & 0 & 0 & 0 & 0 \\
Deadlocking 2 & 0.5036 & 0.4799 & 0.4993 & 0.4536 & 0.4841 \\
Deadlocking 3 & 0.8767 & 0.9095 & 0.8869 & 0.9117 & 0.8962 \\
Fault of $\mathrm{CCL}_{1}$ & 0.9346 & 0.5421 & 0.5326 & 0.5294 & 0.6347 \\
Fault of $\mathrm{CCL}_{2}$ & 0.9410 & 0.9579 & 0.9385 & 0.8669 & 0.9261 \\
Fault of $\mathrm{CCL}_{3}$ & 0.8486 & 0.8358 & 0.8436 & 0.8711 & 0.8498 \\
Fault of $L_{1}$ & 0.8536 & 0.6838 & 0.9414 & 0.7007 & 0.7948 \\
Fault of $L_{2}$ & 0.7620 & 0.5602 & 0.8083 & 0.4979 & 0.6571 \\
Fault of $L_{3}$ & 0.5595 & 0.6189 & 0.8624 & 0.5505 & 0.6478 \\
Fault of $L_{4}$ & 0.6634 & 0.5373 & 0.9156 & 0.8838 & 0.7500 \\
Fault of $D_{1}$ & 0.8407 & 0.9293 & 0.8909 & 0.8907 & 0.8879 \\
Fault of $D_{2}$ & 0.9340 & 0.9502 & 0.9597 & 0.9340 & 0.9445 \\
Fault of $D_{3}$ & 0.9133 & 0.9961 & 0.9106 & 0.9027 & 0.9307 \\
Fault of $D_{4}$ & 0.9241 & 0.9561 & 0.9294 & 0.9390 & 0.9372 \\
\hline
\end{tabular}

3.6. Strategies for Design Improvement. On the basis of the ranking of all the faults shown in Section 3.5, the vital links of the solar array can be found. According to the sorting of the reliability indices, the improvement measures are illustrated in Table 9 [1,27].

\section{Conclusions}

It will prevail that the engineers concern more about the dynamic performance of the mechanical systems. By analyzing the dynamic performance, fault injection aims to quantify the confidence that can be attributed to a system by estimating the consequences of possible faults in the system. It is validated that fault injection is a useful tool for fault severity analysis of the multibody mechanical system, especially for seeking out the week links of the subsystems. The proposed method defined the fault injection technique for simulations and experiments and evaluated the severity by the Gini concordance measure. It can be performed on either simulations and models or working prototypes or systems in the field. The case study illustrated the flow of the new method and demonstrates the correctness of it. In this manner the weaknesses of interactions can be discovered, and the severity ranking of the subsystems provides concrete information of weak parts relatively, which helps the engineers configure the blueprint for reliability design $[29,31]$. For instance, the $\mathrm{CCL}_{1}$ and the locking mechanism $L_{3}$ are the weakest links in the solar array mechanism, which are the useful hints for failure prevention and performance improvement.

Future work include the study of the multifaults and the major issue is to rate the efficiency of the operational behavior of the dependable systems. For one complex mechanical system, the dependencies between all the subsystems and components cannot be ignored. This new research is mainly aimed at providing the estimates for the parameters that usually characterize the operational behavior of the mechanical systems, concerning the intrinsic relations in the system. 
TABLE 9: Strategies for design improvement.

\begin{tabular}{|c|c|c|}
\hline Fault & Ranks of severity & Reliability strategies \\
\hline (1) Deadlocking of the hinges & Deadlocking $1>$ Deadlocking 2 & $\begin{array}{l}\text { Deadlocking is the fatal fault and it is more dangerous if it happens } \\
\text { much earlier. Here are some strategies to get rid of this fault. (1) } \\
\text { Improve the lubrication of the hinges. } \mathrm{MoS}_{2} \text { and graphite are widely } \\
\text { used in the spacecraft as solid lubricant. Other lubricants should be } \\
\text { tested under the environment of space, in order to choose the better } \\
\text { one; (2) improve the sealing device of the hinges }\end{array}$ \\
\hline (2) Fault of CCLs & $\mathrm{CCL}_{1}>\mathrm{CCL}_{3}>\mathrm{CCL}_{2}$ & $\begin{array}{l}\mathrm{CCL}_{2} \text { is the weak link compared with the other CCLs. (1) Use the } \\
\text { cable of new material that is not sensitive to the change of } \\
\text { temperature; (2) use tightener to fasten the cable; (3) design } \\
\text { redundancy for the } \mathrm{CCL}_{2}\end{array}$ \\
\hline $\begin{array}{l}\text { (3) Fault of the locking } \\
\text { mechanism in the hinges }\end{array}$ & $L_{3}>L_{2}>L_{4}>L_{1}$ & $\begin{array}{l}L_{3} \text { is the locking mechanism which tends to fault more easily. The } \\
\text { strategies to improve the reliability of the locking mechanism are } \\
\text { summarized as follows: (1) use the locking spring in } L_{3} \text { with higher } \\
\text { stiffness; (2) use the backup locking springs }\end{array}$ \\
\hline $\begin{array}{l}\text { (4) Fault of the driving torsion } \\
\text { spring }\end{array}$ & $D_{1}>D_{3}>D_{4}>D_{2}$ & $\begin{array}{l}\text { The fault of } D_{1} \text { is the most dangerous. Here are some strategies for } \\
\text { fault prevention: (1) test the torsion spring on the ground, then find } \\
\text { the torque-angle curve to know the characteristics of the torsion } \\
\text { spring more deeply; (2) test the performance of the whole system, } \\
\text { using torsion springs with at least } 20 \% \text { remaining torque }\end{array}$ \\
\hline
\end{tabular}

This research may better indicate the reliability characteristics of the mechanical system.

\section{Conflict of Interests}

The authors declare that there is no conflict of interests regarding the publication of this paper.

\section{Acknowledgments}

This work was supported by the National Science Foundation of China under Contract no. 11272171, Beijing Natural Science Foundation under Contract no. 3132030, and Specialized Research Fund for the Doctoral Program of Higher Education under Contract no. 20120002110070.

\section{References}

[1] D. M. Harland and R. D. Lorenz, Space Systems Faults: Disasters and Rescues of Satellites, Rockets and Space Probes, Springer, Berlin, Germany, 1st edition, 2005.

[2] M. Rausand and A. Høyland, System Reliability Theory, John Wiley \& Sons, New York, NY, USA, 2nd edition, 2004.

[3] J. He and C. Zhang, "A design method for fault reconfiguration and fault-tolerant control of a servo motor," Mathematical Problems in Engineering, vol. 2013, Article ID 647571, 6 pages, 2013.

[4] X. S. Si, W. Wang, C. H. Hu, and D. H. Zhou, "Remaining useful life estimation-a review on the statistical data driven approaches," European Journal of Operational Research, vol. 213, no. 1, pp. 1-14, 2011.

[5] R. Martínez-Guerra, J. L. Mata-Machuca, and J. J. RincónPasaye, "Fault diagnosis viewed as a left invertibility problem," ISA Transactions, vol. 52, no. 5, pp. 652-661, 2013.
[6] W. S. Lee, D. L. Grosh, F. A. Tillman, and C. H. Lie, "Fault tree analysis, methods, and applications: a review," IEEE Transactions on Reliability, vol. 34, no. 3, pp. 194-203, 1985.

[7] N. C. Xiao, Y. F. Li, and H. Z. Huang, "Reliability analysis method of deployment mechanism of a satellite solar arrays," Journal of Astronautics, vol. 30, no. 4, pp. 1704-1710, 2009 (Chinese).

[8] T. Murata, "Petri nets: properties, analysis and applications," Proceedings of the IEEE, vol. 77, no. 4, pp. 541-580, 1989.

[9] C. Y. Yuan, Petri Nets, Press of Southeastern University, Nanjing, China, 1st edition, 1989 (Chinese).

[10] W. Pedrycz and F. Gomide, "Generalized fuzzy Petri net model," IEEE Transactions on Fuzzy Systems, vol. 2, no. 4, pp. 295-301, 1994.

[11] N. G. Leveson and J. L. Stolzy, "Safety analysis using Petri nets," IEEE Transactions on Software Engineering, vol. SE-13, no. 3, pp. 386-397, 1987.

[12] K. W. Chau, "Reliability and performance-based design by artificial neural network," Advances in Engineering Software, vol. 38, no. 3, pp. 145-149, 2007.

[13] M. Llorens, J. Oliver, J. Silva, and S. Tamarit, "Generating a Petri net from a CSP specification: a semantics-based method," Advances in Engineering Software, vol. 50, pp. 110-130, 2012.

[14] Z. F. Bai, Y. Zhao, and H. Tian, "Dynamics simulation of deployment of solar panels in fault modes," Journal of System Simulation, vol. 19, no. 13, pp. 3067-3072, 2007 (Chinese).

[15] O. Wallrapp and S. Wiedemann, "Simulation of deployment of a flexible solar array," Multibody System Dynamics, vol. 7, no. 1, pp. 101-125, 2002.

[16] Airclaims Ascend Space Trak Database, 2014, http://www.seradata.com/spacetrak/Home/Login.aspx.

[17] H. W. Brandhorst Jr. and J. A. Rodiek, "Space solar array reliability: a study and recommendations," Acta Astronautica, vol. 63, no. 11-12, pp. 1233-1238, 2008.

[18] J. Arlat, M. Aguera, L. Amat et al., "Fault injection for dependability validation: a methodology and some applications," IEEE 
Transactions on Software Engineering, vol. 16, no. 2, pp. 166-182, 1990.

[19] M.-C. Hsueh, T. K. Tsai, and R. K. Iyer, "Fault injection techniques and tools," Computer, vol. 30, no. 4, pp. 75-82, 1997.

[20] J. V. Carreira, D. Costa, and J. G. Silva, "Fault injection spotchecks computer system dependability," IEEE Spectrum, vol. 36, no. 8, pp. 50-55, 1999.

[21] A. Benso and P. Prinetto, Fault Injection Techniques and Tools for Embedded Systems Reliability Evaluation, Springer, New York, NY, USA, 1st edition, 2003.

[22] J. M. Voas and G. McGraw, Software Fault Injection: Inoculating Programs against Errors, John Wiley \& Sons, New York, NY, USA, 1st edition, 1997.

[23] R. B. Nelsen, An Introduction to Copulas, Springer, New York, NY, USA, 2nd edition, 2006.

[24] R. B. Nelsen, "Concordance and Gini's measure of association," Journal of Nonparametric Statistics, vol. 9, no. 3, pp. 227-238, 1998.

[25] S. S. Lee, "Noisy replication in skewed binary classification," Computational Statistics and Data Analysis, vol. 34, no. 2, pp. 165-191, 2000.

[26] P. Giudici and E. Raffinetti, "On the Gini measure decomposition," Statistics \& Probability Letters, vol. 81, no. 1, pp. 133-139, 2011.

[27] J. Wu, S. Yan, and L. Xie, "Reliability analysis method of a solar array by using fault tree analysis and fuzzy reasoning Petri net," Acta Astronautica, vol. 69, no. 11-12, pp. 960-968, 2011.

[28] H. A. Linstone and M. Turoff, The Delphi Method: Techniques and Applications, Addison-Wesley, Boston, Mass, USA, 1st edition, 1975.

[29] P. Liu, Fundamental of Reliability Engineering, China Measurement Press, Beijing, China, 1st edition, 2002 (Chinese).

[30] R. E. Larson and J. L. Casti, Principles of Dynamic Programming: Advanced Theory and Applications, Marcel Dekker, New York, NY, USA, 1st edition, 1982.

[31] J. N. Wu, S. Z. Yan, L. Y. Xie et al., "Reliability apportionment approach for spacecraft solar array using fuzzy reasoning Petri net and fuzzy comprehensive evaluation," Acta Astronautica, vol. 76, pp. 136-144, 2012. 


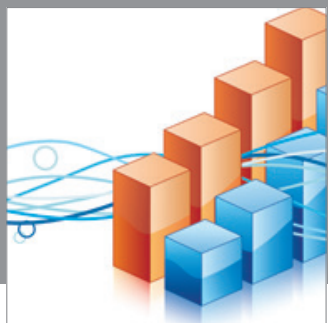

Advances in

Operations Research

mansans

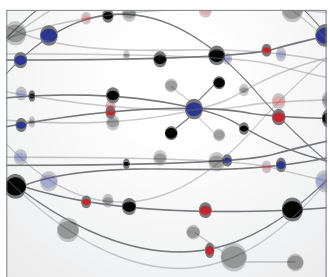

The Scientific World Journal
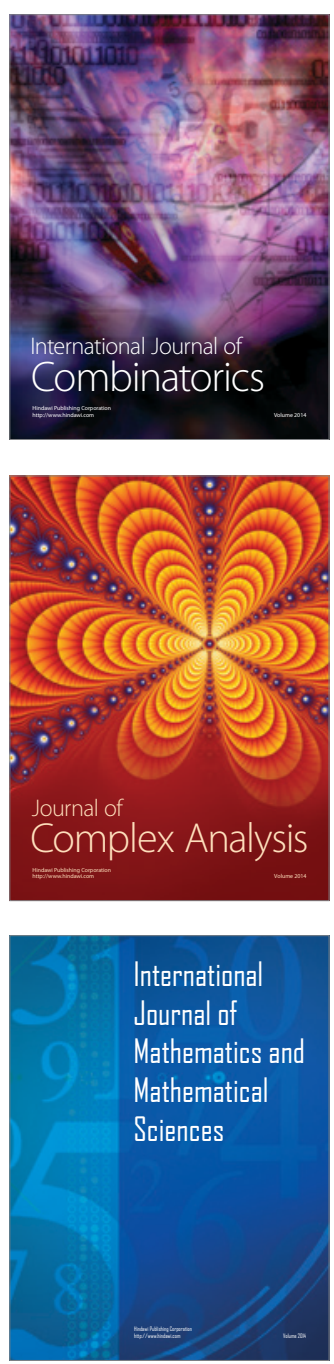
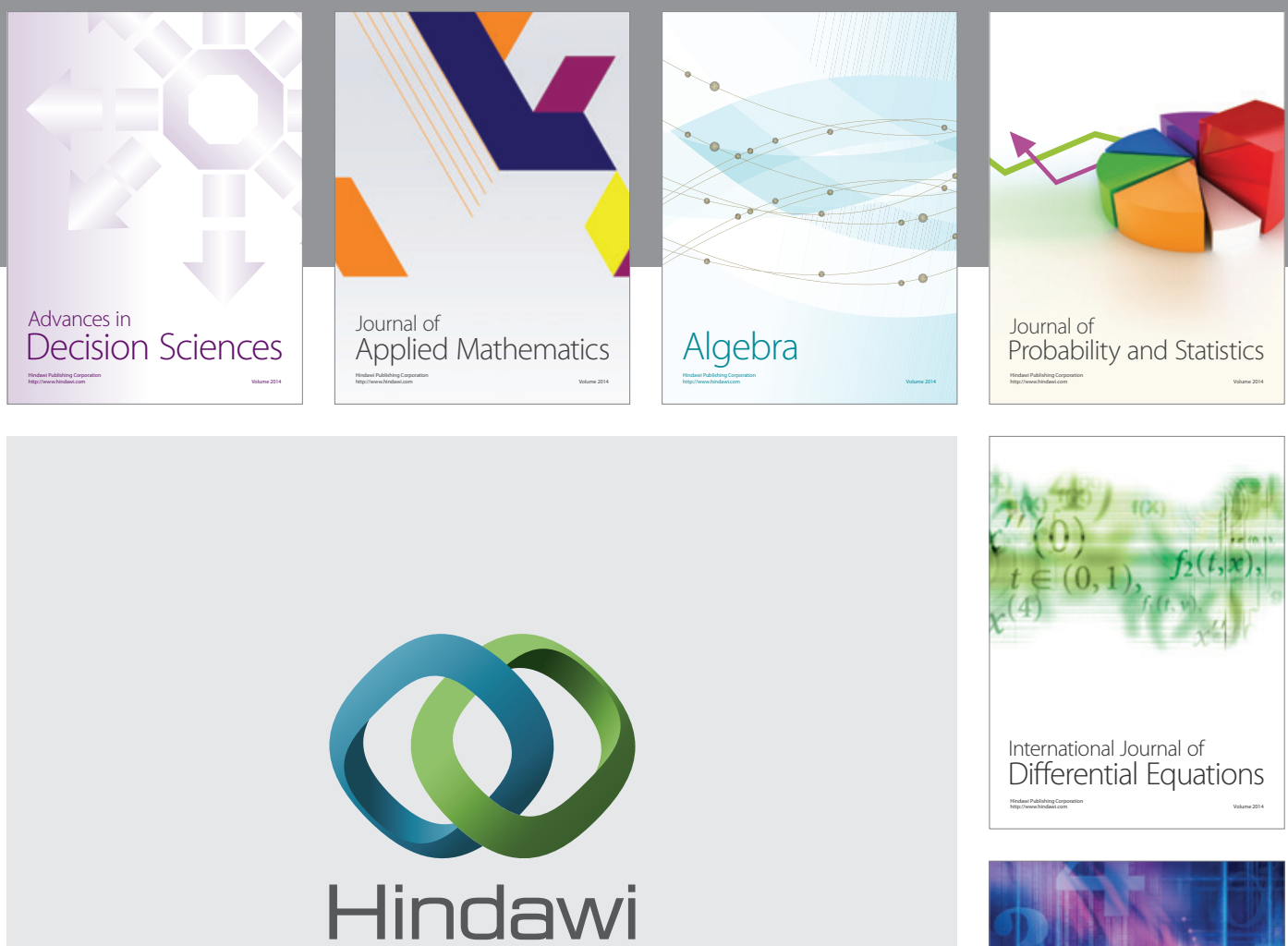

Submit your manuscripts at http://www.hindawi.com
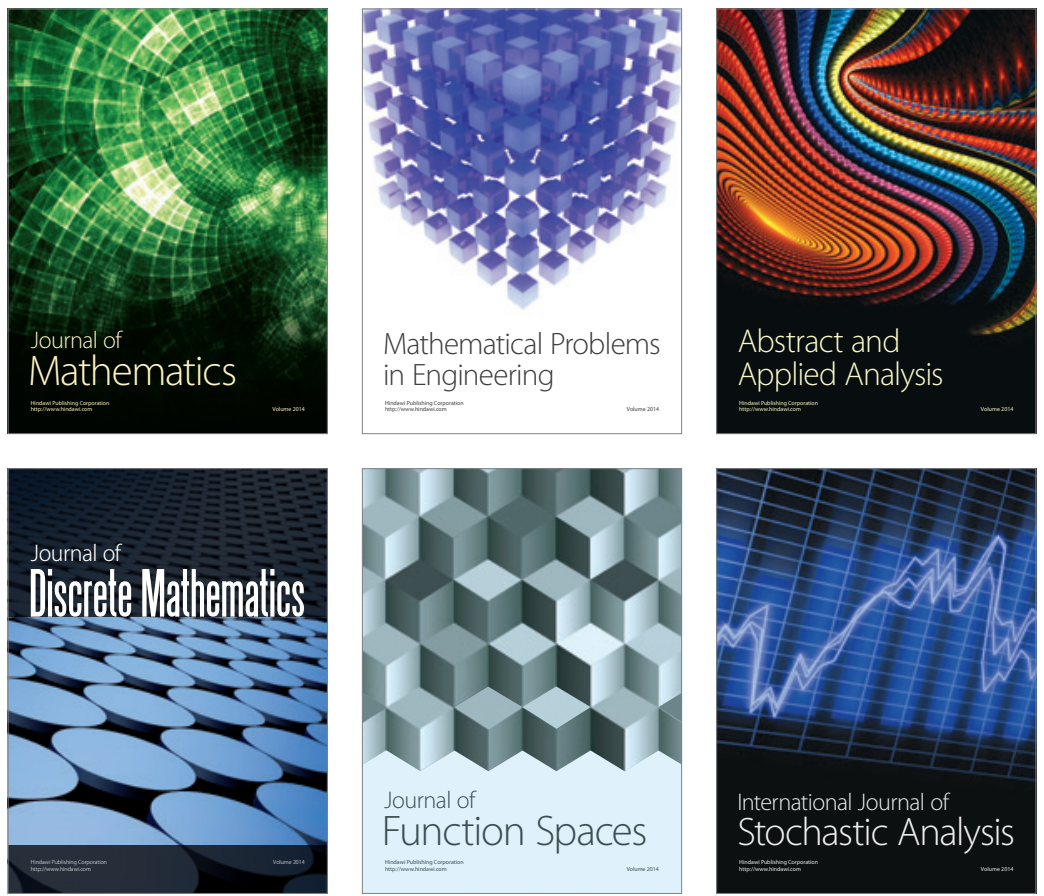

Journal of

Function Spaces

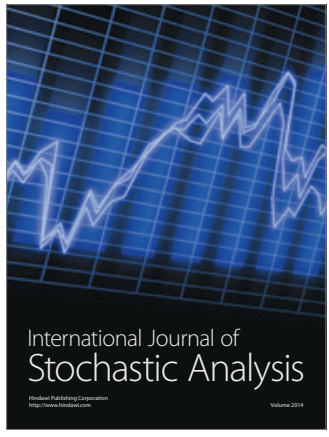

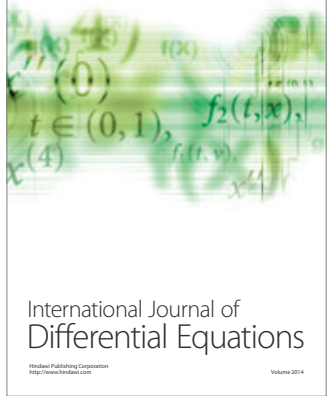
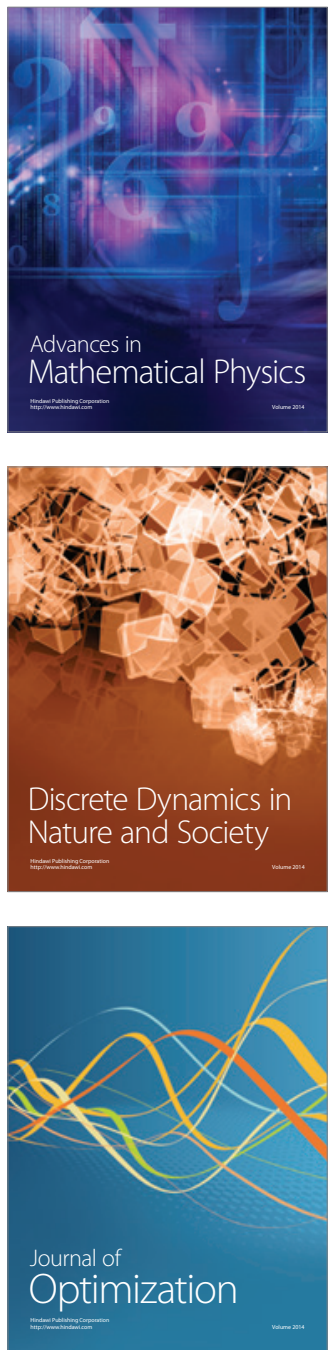